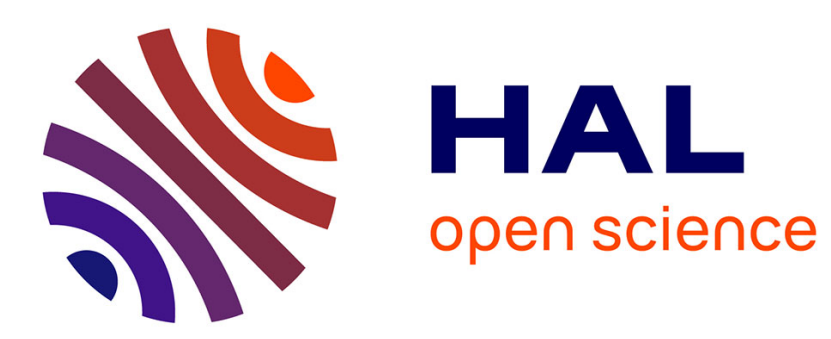

\title{
Use of downscaling procedure for macroscopic heat source modeling in porous media
}

\author{
Vincent Leroy, Dominique Bernard
}

\section{To cite this version:}

Vincent Leroy, Dominique Bernard. Use of downscaling procedure for macroscopic heat source modeling in porous media. Transport in Porous Media, 2018, 122 (2), pp.459-486. 10.1007/s11242-0181013-6 . hal-01742089

\author{
HAL Id: hal-01742089 \\ https://hal.science/hal-01742089
}

Submitted on 23 Mar 2018

HAL is a multi-disciplinary open access archive for the deposit and dissemination of scientific research documents, whether they are published or not. The documents may come from teaching and research institutions in France or abroad, or from public or private research centers.
L'archive ouverte pluridisciplinaire HAL, est destinée au dépôt et à la diffusion de documents scientifiques de niveau recherche, publiés ou non, émanant des établissements d'enseignement et de recherche français ou étrangers, des laboratoires publics ou privés. 


\title{
Use of a downscaling procedure for macroscopic heat source modeling in porous media
}

\author{
Vincent Leroy ${ }^{1,2,3}$ and Dominique Bernard ${ }^{* 1,2}$ \\ ${ }^{1}$ CNRS, Institut de chimie de la matière condensée de Bordeaux, UMR 5026, 33600 \\ Pessac, France \\ ${ }^{2}$ Université de Bordeaux, Institut de chimie de la matière condensée de Bordeaux, \\ UMR 5026, 33600 Pessac, France \\ ${ }^{3}$ Rayference, rue d'Alost 7-11, 1000 Bruxelles, Belgium
}

\begin{abstract}
This work deals with the challenges brought by nonlinear heat sources when upscaling heat transfer in porous media. These difficulties are exemplified through applying the volume averaging method to a simple convective heat transfer problem in a porous medium featuring a nonlinear heterogeneous heat source. The most general solution proposed requires the availability of an estimated value of the heat source in the averaging volume, which can be obtained through a multiscale approach making use of a downscaling methodology. The downscaling methodology yields pore-scale governing equations in a sub-domain of the porous medium allowing to deal with the lack of information about the thermal behavior of the sub-domain's vicinity. Solving the downscaled equations allows to reconstruct the temperature field and the heat source in the sub-domain with a good accuracy. This approximated reconstruction of the temperature field in sub-domain makes it possible to compute accurate estimates of the macroscopic heat source. In practice, the computational cost of the multiscale approach can be reduced by storing the results of the downscaling procedure in a table which takes as entries the limited number of macro-scale dependencies of the downscaled problem's solution. In an example, the resulting heat source table is used as an input in a heuristic macro-scale transport model and compared to classic approaches. The use of the heat source reconstructed by downscaling results in a significant improvement of the accuracy of the macro-scale solution when the temperature driving the heat source significantly deviates from the macro-scale temperature.
\end{abstract}

Keywords: Heat transfer $\bullet$ Porous medium $\bullet$ Nonlinearity Downscaling • Upscaling • Volume averaging

*dominique.bernard@icmcb-bordeaux.cnrs.fr 


\section{List of symbols}

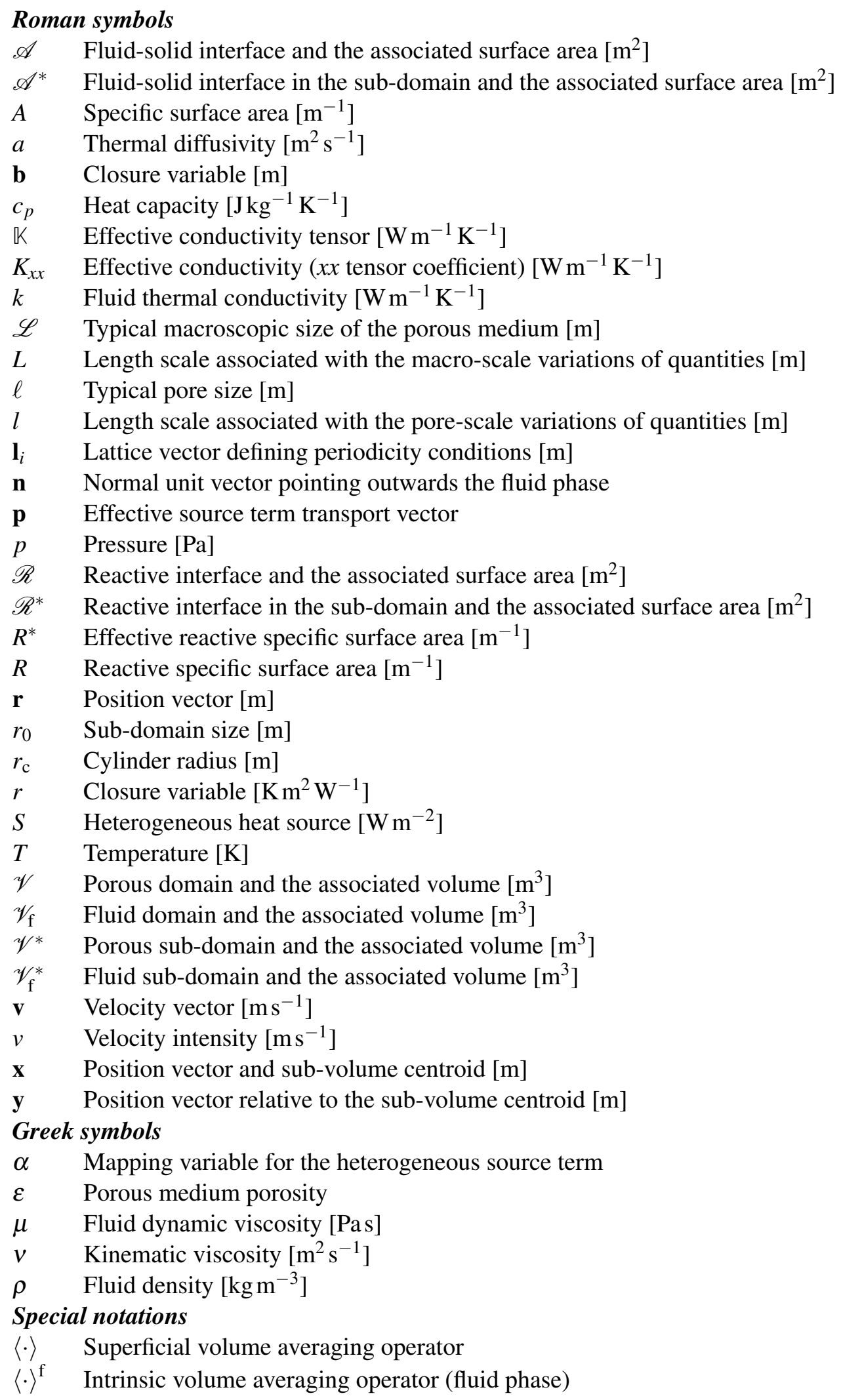




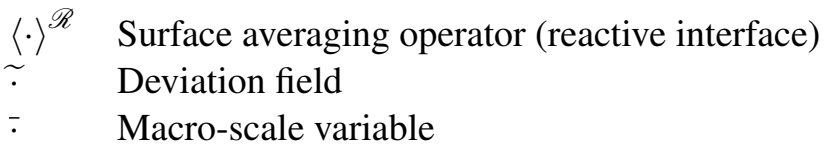

\section{Introduction}

Heat transfer in porous media has a key role in many applications. A lot of them involve phenomena associated with highly nonlinear physics (e.g. combustion, radiation transfer, pyrolysis, catalysis, etc.) and are a constant source of questioning when applying upscaling techniques. In most cases, approximations have to be used, and the resulting macro-scale transport model is always a compromise between complexity and accuracy. Many studies have dealt with nonlinear heat transfer problems in porous media and have come to various conclusions and solutions, some of which are summarized by Quintard (2014).

Even when the usual length-scale constraints are satisfied, i.e. in the absence of "shocks" in macroscopic quantities (e.g. combustion fronts, thermal contact, interfaces, etc.), homogeneous and heterogeneous source term nonlinearity remains a challenge in many applications. It is often handled through partial decoupling of pore-scale physics (Whitaker, 1998; Quintard and Whitaker, 2000). However, in some situations, pore-scale physics coupling remains important and finer estimates of source terms would be a significant improvement. A porescale approximate of the temperature field in a volume representative of the medium could be used to compute a better estimate of source terms. Nevertheless, as repeatedly mentioned in the upscaling literature, performing direct numerical simulation (DNS) in the entire porous medium is impossible due to computational costs, and doing it in a sub-volume of the macroscopic system is non-trivial due to missing information about the vicinity of the sample.

Angeli et al. (2013) proved that a downscaling procedure can yield an approximate of velocity and temperature fields in a sub-volume of the system with good accuracy in the case of an incompressible, anisothermal flow with a constant heterogeneous heat source. This approach compensates for the lack of information about the sub-volume's vicinity by using macroscopic field variations and periodic boundary conditions. In this work, the methodology is extended to cases with heterogeneous source terms nonlinearly depending on temperature. If the resulting approximate temperature field is accurate enough, it can be used to retrieve an estimate of the average heterogeneous source term in the sub-volume. Downscaled equations have clearly identified macroscopic dependencies and their solutions can therefore be easily tabulated against a reduced number of macroscopic variables. The resulting table can then be used to compute macroscopic source terms. Such a methodology could represent a significant improvement in the computation of macroscopic source terms.

In Sect. 2, we highlight major issues brought by nonlinearity when applying the volume averaging method (Whitaker, 1999) to a simple incompressible, anisothermal flow problem with a heterogeneous source term featuring an unspecified dependency against temperature. We propose an original solution to these issues implying the computation of an estimate of the macro-scale source term using a downscaling technique. In Sect. 3, we present the downscaling procedure used to construct an approximate temperature field in a sub-volume of the system. The resulting pore-scale solution is compared to a reference solution obtained by direct numerical simulation and the accuracy of the methodology is assessed. In Sect. 4, we apply the multiscale methodology proposed in Sect. 2 to an example and use the downscaling methodology to compute the macro-scale source term in a heuristic macro-scale heat transfer 
model. The results are compared with the solution obtained when using a simpler macro-scale heat source model.

\section{Upscaling of a nonlinear heat transfer problem}

In this section, difficulties brought by nonlinearity of heterogeneous source terms when performing volume averaging are highlighted using a simple example. The volume averaging method applied to heat transfer problems was covered in-depth for a variety of configurations (Quintard and Whitaker, 1993; Moyne, 1997; Quintard et al., 1997; Whitaker, 1998). The present section follows the ideas presented by Carbonell and Whitaker (1983) and Quintard and Whitaker (2000). The example which, despite its physically unrealistic nature, includes all the features necessary for the analysis while remaining as simple as possible for clarity.

It should be noted that for best consistency with the averaging procedure used in Sect. 3.2, the averaging filter should be defined using weighting functions and a convolution product (Marle, 1967; Quintard and Whitaker, 1993). However, for clarity, the classic approach will be used in these developments; this corresponds to the use of a top-hat weighting function.

\subsection{Governing equations}

We consider the steady-state, incompressible, anisothermal flow of a fluid in a spatially homogeneous porous medium (see Fig. 1). The volume of the system is denoted $\mathscr{V}$ and the volume occupied by the fluid phase is denoted $\mathscr{V}_{\mathrm{f}}$. The fluid-solid interface is denoted $\mathscr{A}$ and the surface normal unit vector pointing outwards the fluid phase is denoted $\mathbf{n}$. In the following, $\mathbf{r}$ will be a point in the medium. The pore-scale flow governing equations and boundary conditions are:

$$
\begin{aligned}
\nabla \cdot \mathbf{v} & =0 & & \text { in } \mathscr{V} / \mathrm{f} \\
\nabla \cdot(\rho \mathbf{v v}) & =\mu \nabla^{2} \mathbf{v}-\nabla p & & \text { in } \mathscr{V} / \mathrm{f} \\
\mathbf{v} & =\mathbf{0} & & \text { at } \mathscr{A}
\end{aligned}
$$

where $\mathbf{v}$ and $p$ are respectively the velocity and pressure fields in the fluid phase, and $\mu$ and $\rho$ are respectively the dynamic viscosity and density of the fluid phase. Boundary conditions at $\partial \mathscr{V}_{\mathrm{f}} \backslash \mathscr{A}$, although mathematically required for the problem to be well-posed, are not specified because they will not be used in this section.

The pore-scale governing energy equation and boundary conditions are:

$$
\begin{aligned}
\nabla \cdot\left(\rho c_{p} \mathbf{v} T\right) & =\nabla \cdot(k \nabla T) & & \text { in } \mathscr{V}_{\mathrm{f}} \\
\mathbf{n} \cdot k \nabla T & =S(T) & & \text { at } \mathscr{R} \\
\mathbf{n} \cdot k \nabla T & =0 & & \text { at } \mathscr{A} \backslash \mathscr{R}
\end{aligned}
$$

where $T$ is the temperature field in the fluid phase and $c_{p}$ and $k$ are respectively the heat capacity and thermal conductivity of the fluid phase. $S(T)$ is a heterogeneous source term, arbitrarily varying as a function of temperature. $\mathscr{R}$ is the part of $\mathscr{A}$ designated as the reactive interface.

It should be noted that in practical cases, the solid phase is more likely to be closer to the infinite conductivity limit than to the null conductivity one. However, despite its unrealistic nature, this set of governing equations highlights the challenges of nonlinear source terms while remaining simple. Moreover, it shares many similarities with quasi-steady reactive mass transport problems. 


\subsection{Volume averaging}

The volume averaging operator is applied to the governing equations, using, as the averaging volume, a sub-domain $\mathscr{V}^{*}(\mathbf{x})$ centered at $\mathbf{x} . \mathscr{V}_{\mathrm{f}}^{*}(\mathbf{x})$ is the part of $\mathscr{V}^{*}(\mathbf{x})$ occupied by the fluid phase. Similarly, $\mathscr{A}^{*}(\mathbf{x})$ is $\mathscr{A} \cap \mathscr{V}^{*}(\mathbf{x})$. For simplicity, a sub-domain (resp. sub-surface) and its volume (resp. surface area) will be identified in the following. The characteristic size of $\mathscr{V}^{*}$ is denoted $r_{0}$. Following the usual volume averaging practices, we define the superficial and intrinsic averages, related by the porosity $\varepsilon$, as

$$
\begin{aligned}
\langle\Psi\rangle(\mathbf{x}) & =\frac{1}{\mathscr{V}^{*}(\mathbf{x})} \int_{\mathscr{V}_{\mathrm{f}}^{*}(\mathbf{x})} \Psi(\mathbf{r}) \mathrm{d} \mathscr{V}(\mathbf{r}) \\
\langle\Psi\rangle^{\mathrm{f}}(\mathbf{x}) & =\frac{1}{\mathscr{V}_{\mathrm{f}}^{*}(\mathbf{x})} \int_{\mathscr{V}_{\mathrm{f}}^{*}(\mathbf{x})} \Psi(\mathbf{r}) \mathrm{d} \mathscr{V}(\mathbf{r})=\varepsilon\langle\Psi\rangle(\mathbf{x})
\end{aligned}
$$

where $\Psi$ is a physical field (scalar or vector), $\mathbf{x}$ the centroid of $\mathscr{V}^{*}(\mathbf{x})$ and $\mathbf{r}$ a current point in the averaging volume. In the following, spatial dependencies will be omitted for conciseness when unambiguous. Use will be made of the spatial decomposition (Gray, 1975):

$$
\Psi(\mathbf{r})=\langle\Psi\rangle^{\mathrm{f}}(\mathbf{r})+\widetilde{\Psi}(\mathbf{r})
$$

and the spatial averaging theorems:

$$
\begin{aligned}
\langle\nabla \cdot \Psi\rangle & =\nabla \cdot\langle\Psi\rangle+\frac{1}{\mathscr{V}^{*}} \int_{\mathscr{A}^{*}} \mathbf{n} \cdot \Psi \mathrm{d} \mathscr{A} \\
\langle\nabla \Psi\rangle & =\nabla\langle\Psi\rangle+\frac{1}{\mathscr{V}^{*}} \int_{\mathscr{A}^{*}} \mathbf{n} \Psi \mathrm{d} \mathscr{A}
\end{aligned}
$$

The length-scale constraints and order of magnitude estimates implied by the procedure are extensively discussed in the volume averaging method literature (see Appendix A). They can, in many common cases, be ensured by if scale separation is valid (Whitaker, 1969), i.e. if

$$
l \ll r_{0} \ll L
$$

In Eq. (8), $l$ is the largest length scale associated with the variation of pore-scale quantities, and $L$ is the smallest length scale associated with the variations of macro-scale quantities. $l$ and $L$ are usually consistent with the quantities $\ell$ and $\mathscr{L}$ defined in Fig. 1 . The application of the averaging procedure to flow equations has been covered in-depth (Whitaker, 1986, 1996) and will consequently not be repeated here.

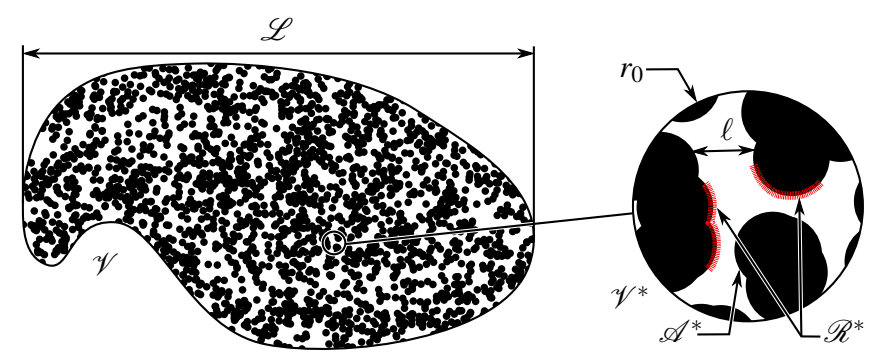

Figure 1: Porous medium and length scales; the fluid phase is represented in white. 
When applying the averaging operator to Eq. (2a), we get:

$$
\begin{aligned}
\rho c_{p} \nabla \cdot\left(\varepsilon\langle\mathbf{v}\rangle^{\mathrm{f}}\langle T\rangle^{\mathrm{f}}\right) & \\
= & \nabla \cdot\left(\varepsilon k \nabla\langle T\rangle^{\mathrm{f}}\right)+\nabla \cdot\left(\frac{k}{\mathscr{V}^{*}} \int_{\mathscr{A}^{*}} \mathbf{n} \widetilde{T} \mathrm{~d} \mathscr{A}\right)+\frac{1}{\mathscr{V}^{*}} \int_{\mathscr{A}^{*}} \mathbf{n} \cdot k \nabla \widetilde{T} \mathrm{~d} \mathscr{A}
\end{aligned}
$$

The following operations are then performed:

- subtract Eq. (9) from Eq. (2a) multiplied by $\varepsilon$ and use order of magnitude estimates to get a governing equation for the deviation field $\widetilde{T}$;

- insert the spatial decomposition into boundary condition (2b);

- apply periodic boundary conditions on $\mathscr{V}^{*}$, which means that $\mathscr{V}^{*}$ is assumed to be representative of the medium;

- apply the null average constraint for the deviation field.

This allows us to write the deviation problem:

$$
\begin{aligned}
\rho c_{p}\left(\nabla \cdot(\widetilde{\mathbf{v}} \widetilde{T})+\nabla \cdot\left(\mathbf{v}\langle T\rangle^{\mathrm{f}}\right)\right) & =\nabla \cdot(k \nabla \widetilde{T})-\frac{1}{\mathscr{V}_{\mathrm{f}}^{*}} \int_{\mathscr{A}^{*}} \mathbf{n} \cdot k \nabla \widetilde{T} \mathrm{~d} \mathscr{A} & & \text { in } \mathscr{V}_{\mathrm{f}}^{*} \\
\mathbf{n} \cdot k \nabla \widetilde{T} & =-\mathbf{n} \cdot k \nabla\langle T\rangle^{\mathrm{f}}+S(T) & & \text { at } \mathscr{R}^{*} \\
\mathbf{n} \cdot k \nabla \widetilde{T} & =-\mathbf{n} \cdot k \nabla\langle T\rangle^{\mathrm{f}} & & \text { at } \mathscr{A}^{*} \backslash \mathscr{R}^{*} \\
\widetilde{T}\left(\mathbf{r}+\mathbf{l}_{i}\right) & =\widetilde{T}(\mathbf{r}) & & i \in \llbracket 1,3 \rrbracket \\
\langle T\rangle & =0 & &
\end{aligned}
$$

where the $\mathbf{l}_{i}$ are lattice vectors defining periodicity conditions. It is worth noticing that in Eqs. (10), the macro-scale quantities are evaluated at the centroid $\mathbf{x}$ of $\mathscr{V}^{*}$, which requires length-scale constraints to be satisfied and ensures compliance with the periodicity conditions. At this stage, $S(T)$ is not explicitly written in terms of dependencies versus $\langle T\rangle^{\mathrm{f}}$ and $\widetilde{T}$. It however has to be a periodic function of $\mathbf{r}$ to be compatible with the periodicity conditions.

In order to proceed with the analysis, writing $S$ as a function of macro-scale dependencies is essential. Two strategies are possible:

1. A first approach consists in writing an explicit dependency versus $\langle T\rangle^{\mathrm{f}}$. In that case, in order to keep periodicity, the estimate for $S(T)$ has to be periodic. Therefore, we will write:

$$
S(T) \simeq S\left(\langle T\rangle^{\mathrm{f}}(\mathbf{x})+\widetilde{T}\right)
$$

This approximation is valid under specific length-scale constraints, which are determined through a Taylor series development of $S$ around $\langle T\rangle^{\mathrm{f}}(\mathbf{x})+\widetilde{T}$ and an order of magnitude analysis. This is very similar to the procedure leading to the downscaled boundary condition in Sect. 3.1 (see also Appendix B). We then use another Taylor series development of the source term around $\langle T\rangle^{\mathrm{f}}(\mathbf{x})$ :

$$
\begin{aligned}
S\left(\langle T\rangle^{\mathrm{f}}(\mathbf{x})+\right. & \widetilde{T}) \\
& =S\left(\langle T\rangle^{\mathrm{f}}(\mathbf{x})\right)+\widetilde{T} \frac{\mathrm{d} S}{\mathrm{~d} T}\left(\langle T\rangle^{\mathrm{f}}(\mathbf{x})\right)+\frac{1}{2} \widetilde{T}^{2} \frac{\mathrm{d}^{2} S}{\mathrm{~d}^{2}}\left(\langle T\rangle^{\mathrm{f}}(\mathbf{x})\right)+\ldots
\end{aligned}
$$


A zeroth-order truncation is common practice (Quintard and Whitaker, 2005) and is appropriate if the temperature at $\mathscr{R}^{*}$ does not significantly deviate from $\langle T\rangle^{\mathrm{f}}(\mathbf{x})$. Stopping at the first order (Whitaker, 1998; Guo et al., 2015), it is then possible to account for $T$ deviating from $\langle T\rangle^{\mathrm{f}}(\mathbf{x})$, at the cost of additional complexity in both the macroscopic problem and the closure process. Moreover, it can be inaccurate if $S$ has a highly nonlinear behavior around $\langle T\rangle^{\mathrm{f}}(\mathbf{x})$. Finally, going up to an order higher than the first introduces $\widetilde{T}^{2}$, which is impractical given the linear nature of the closure relations used. Therefore, in cases where $S$ is strongly nonlinear and the interface temperature strongly deviates from the average temperature, the Taylor series expansion strategy falls short.

2. Another possible decomposition would be

$$
S=\langle S\rangle^{\mathscr{R}}+\widetilde{S}
$$

where

$$
\langle\Psi\rangle^{\mathscr{R}}=\frac{1}{\mathscr{R}^{*}} \int_{\mathscr{R}^{*}} \Psi \mathrm{d} \mathscr{A}
$$

In that case, the average value $\langle S\rangle^{\mathscr{R}}$ is the average heterogeneous source term at the interface. This decomposition is relevant since $\langle S\rangle^{\mathscr{R}}$ naturally emerges when writing the closed-form averaged equations. The challenge in estimating $\widetilde{S}$ often leads authors to neglect this deviation component (Quintard et al., 2000). However, the analysis can be continued by writing $S$ in the form

$$
S \simeq\langle S\rangle^{\mathscr{R}}(1+\alpha)
$$

where $\alpha$ is a periodic function estimated from $\widetilde{S} /\langle S\rangle^{\mathscr{R}}$. Leroy et al. (2013) applied this strategy and concluded that an iterative procedure is required to solve the resulting closure problems. This is true if $\alpha$ is supposed unknown when solving the closure problems.

When following the second approach, the deviation problem (10) becomes:

$$
\begin{aligned}
\rho c_{p}\left(\nabla \cdot(\widetilde{\mathbf{v}})+\nabla \cdot\left(\mathbf{v}\langle T\rangle^{\mathrm{f}}\right)\right) & =\nabla \cdot(k \nabla \widetilde{T})-\frac{1}{\mathscr{V}_{\mathrm{f}}^{*}} \int_{\mathscr{A}^{*}} \mathbf{n} \cdot k \nabla \widetilde{T} \mathrm{~d} \mathscr{A} & & \text { in } \mathscr{V}_{\mathrm{f}}^{*} \\
\mathbf{n} \cdot k \nabla \widetilde{T} & =-\mathbf{n} \cdot k \nabla\langle T\rangle^{\mathrm{f}}+\langle S\rangle^{\mathscr{R}}(1+\alpha) & & \text { at } \mathscr{R}^{*} \\
\mathbf{n} \cdot k \nabla \widetilde{T} & =-\mathbf{n} \cdot k \nabla\langle T\rangle^{\mathrm{f}} & & \text { at } \mathscr{A}^{*} \backslash \mathscr{R}^{*} \\
\widetilde{T}\left(\mathbf{r}+\mathbf{l}_{i}\right) & =\widetilde{T}(\mathbf{r}) & & i \in \llbracket 1,3 \rrbracket \\
\langle T\rangle & =0 & &
\end{aligned}
$$

Macroscopic source terms are then identified and the following closure relation is proposed:

$$
\widetilde{T}=\mathbf{b} \cdot \nabla\langle T\rangle^{\mathrm{f}}+r\langle S\rangle^{\mathscr{R}}
$$

Closure problems resulting from this relation are presented in Appendix C.

Use of closure (17) in Eq. (9) yields the macroscopic closed equation

$$
\rho c_{p} \nabla \cdot\left(\varepsilon\langle\mathbf{v}\rangle^{\mathrm{f}}\langle T\rangle^{\mathrm{f}}\right)=\nabla \cdot\left(\mathbb{K} \cdot \nabla\langle T\rangle^{\mathrm{f}}\right)+R^{*}\langle S\rangle^{\mathscr{R}}+\nabla \cdot\left(\mathbf{p}\langle S\rangle^{\mathscr{R}}\right)
$$


where $R^{*}$ is the effective reactive specific surface area, given by

$$
R^{*}=\frac{1}{\mathscr{V}^{*}} \int_{\mathscr{R}^{*}}(1+\alpha) \mathrm{d} \mathscr{A}
$$

$\mathbb{K}$ is the effective conductivity tensor, given by

$$
\mathbb{K}=\varepsilon k \rrbracket+\frac{k}{\mathscr{V}^{*}} \int_{\mathscr{A}^{*}} \mathbf{n b} \mathrm{d} \mathscr{A}-\rho c_{p}\langle\widetilde{\mathbf{v}} \mathbf{b}\rangle
$$

and $\mathbf{p}$ is a dimensionless vector given by

$$
\mathbf{p}=\frac{k}{\mathscr{V}^{*}} \int_{\mathscr{A}^{*}} \mathbf{n} r \mathrm{~d} \mathscr{A}-\rho c_{p}\langle\widetilde{\mathbf{v}} r\rangle
$$

While providing a general interpretation of $\mathbf{p}$ in terms of macroscopic physics is difficult due to the generic form chosen for $S$, it is interesting to note that this effective property shares many similarities with the non-traditional velocity introduced by Guo et al. (2015), from its expression to the closure problem which allows its computation. Since the values of $R^{*}$ and $\mathbf{p}$ depend on $\alpha$, itself depending on the expression of $S$ and the macroscopic context (i.e. the thermal behavior of the macroscopic system), estimating these quantities requires a dedicated study, beyond the scope of this work.

It then becomes obvious that neglecting $\widetilde{S}$ is equivalent to neglecting $\alpha$, i.e. considering that the flux at $\mathscr{R}^{*}$ deviates from its average in a negligible way.

From this brief overview of the upscaling procedure, we can see that source term nonlinearity introduces challenges. Even when using the $\alpha=0$ approximation, an estimate of $\langle S\rangle^{\mathscr{R}}$ remains necessary to perform macroscopic heat transfer predictions (see Eq. (18)). When the interface temperature is close to $\langle T\rangle^{\mathrm{f}}$, using

$$
\langle S\rangle^{\mathscr{R}} \simeq S\left(\langle T\rangle^{\mathrm{f}}\right)
$$

is sensible (Quintard and Whitaker, 2005) (and equivalent to the zeroth-order Taylor series expansion strategy); however, when the interface temperature significantly deviates from $\langle T\rangle^{\mathrm{f}}$, estimating $\langle S\rangle^{\mathscr{R}}$ becomes more challenging, because it requires an estimate of the interface temperature at the pore scale.

Running direct numerical simulation with governing equations in $\mathscr{V}$ to extract the resulting temperature field in $\mathscr{V}^{*}$ is unfeasible due to the excessive amount of computational resources required: $\langle S\rangle^{\mathscr{R}}$ should therefore be estimated only for the volume $\mathscr{V}^{*}$. The major difficulty is that the boundary conditions at $\partial \mathscr{V}_{\mathrm{f}}^{*} \backslash \mathscr{A}^{*}$ are unspecified and impossible to determine due to the lack of information about the vicinity of $\mathscr{V}^{*}$ (it is also the reason why periodic boundary conditions had to be used in Sect. 2.2). However, macro-scale fields (e.g. $\langle T\rangle^{\mathrm{f}}$ ) contain a large amount of information which can be used to model the pore-scale behavior of the temperature field in $\mathscr{V}^{*}$. For that purpose, a downscaling methodology can be used.

\subsection{Multiscale methodology}

Before discussing the downscaling procedure, it is important to see how it can be used in the framework of a multiscale algorithm. The macro-scale governing equation (18) results from an upscaling procedure, which models the influence of pore-scale phenomena at the 
macroscopic scale. From the final developments in Sect. 2.2, we can conclude that the macroscopic scale has an influence on the pore scale, and that this can be modeled with a downscaling methodology. The macro-scale and pore-scale (downscaled) models can therefore be coupled to yield a multiscale model, which will account for multiscale coupling effects with improved accuracy.

In a multiscale approach, some terms in the macro-scale governing equations (e.g. $\langle S\rangle^{\mathscr{R}}$ in Eq. (18)) depend on the results of a downscaled model. On the other side, the results of the downscaled model depend on the macro-scale behavior of the system: both must therefore be solved in a coupled way. A possible coupling strategy relies on an iterative approach:

1. A solution to the macro-scale governing equation (18) is computed using an estimate of $\langle S\rangle^{\mathscr{R}}$.

2. The resulting macro-scale information is used as the input of a downscaled model to compute a better estimate for $\langle S\rangle^{\mathscr{R}}$ at a reasonable computational cost.

3. The new estimate for $\langle S\rangle^{\mathscr{R}}$ is used to solve Eq. (18) again.

4. Steps 2 and 3 are repeated until the macro-scale solution has converged.

This strategy is further discussed, refined and applied to an example in Sect. 4.

Various multiscale approaches have been developed for the simulation of transport in porous media, notably in the reservoir simulation community (Arbogast et al., 2007; Chen et al., 2003; Fredrik and Tveito, 1998; Gautier et al., 1999; Hou and Wu, 1997; Jenny et al., 2005; Juanes, 2005). However, these methods start from an already homogenized description of the medium at the finer scale and mainly aim at dealing with difficulties caused by strong, short-scale variations of homogenized transport properties, such as permeability. The methodology applied in this work, based on volume averaging and the work of Angeli et al. (2013), is based on a non-homogenized (discrete) pore-scale description of the medium and serves a different purpose.

\section{Downscaling procedure}

Downscaling is a class of modeling techniques aiming at inferring the micro-scale behavior of a system from only macro-scale information. This macro-scale information can originate from experimental data, or it can be obtained from macro-scale transport models, built using phenomenological upscaling approaches, solved analytically or numerically. Downscaling is extensively used for climate modeling and meteorology (Von Storch et al., 1993; Wilby et al., 1998; Maraun et al., 2010), and has been mentioned as part of multiscale strategies for reservoir simulation (Babaei and King, 2012). Angeli et al. (2013) developed a downscaling procedure based on the volume averaging method. In the following, we apply the method to the problem presented in Sect. 2 .

Starting from a reference problem, whose solution is a reference field $\Psi$ (where $\Psi$ is $\mathbf{v}$ or $T$ ) defined in $\mathscr{V}$, we build a cell problem, whose solution is an approximate $\Psi^{*}$ of $\Psi$, defined in $\mathscr{V}^{*} . \Psi^{*}$ is required to satisfy governing equations as close as possible to those governing $\Psi$. It is assumed that if there are small enough differences between the problems from which $\Psi$ and $\Psi^{*}$ are respectively solutions, then $\Psi$ and $\Psi^{*}$ will be close.

The cell problem is developed under several assumptions, which will allow to compensate for the information deficit about the sub-volumes neighboring $\mathscr{V}^{*}$, and it uses macro-scale 
data as an input. In the present case, the reference problem is described in Sect. 2.1 with sufficient thoroughness to perform downscaling. The validation of the downscaling methodology is performed using the approach of Angeli et al. (2013): a reference solution (which will require the specification of the geometry of the medium and the boundary conditions at $\left.\partial \mathscr{V}_{\mathrm{f}} \backslash \mathscr{A}\right)$ is compared to a downscaled solution.

\subsection{Cell problem}

In the following, it will be assumed that the porous medium is homogeneous in the vicinity of $\mathscr{V}^{*}$, i.e. that

$$
\nabla \varepsilon(\mathbf{r})=\mathbf{0} \quad \text { for } \mathbf{r} \in \mathscr{V}^{*}(\mathbf{x})
$$

The development of the cell problem is based on the use of decomposition (5) in reference governing equations. The macroscopic field $\langle T\rangle^{\mathrm{f}}$ is assumed to be known in $\mathscr{V}^{*}$. Following the common practice in volume averaging, we will compensate the lack of information about the neighboring sub-volumes by assuming that the deviation component of our approximated field is periodic. Therefore, the reconstructed field $\Psi^{*}$ will be approximated by

$$
\Psi^{*}(\mathbf{r})=\langle\Psi\rangle^{\mathrm{f}}(\mathbf{r})+\widetilde{\Psi}^{*}(\mathbf{r})
$$

where $\widetilde{\Psi}^{*}$ is periodic over $\mathscr{V}^{*}$ :

$$
\widetilde{\Psi}^{*}\left(\mathbf{r}+\mathbf{l}_{i}\right)=\widetilde{\Psi}^{*}(\mathbf{r})
$$

The relevance of periodic boundary conditions can be discussed as in the case of upscaling.

When performing the upscaling process, it can be shown that the $\widetilde{\Psi}$ fields have to comply with the null average constraint:

$$
\langle\widetilde{\Psi}\rangle(\mathbf{x})=0
$$

We will therefore impose

$$
\left\langle\widetilde{\Psi}^{*}\right\rangle(\mathbf{x})=0
$$

It should be kept in mind that both the periodic boundary conditions and the null-average constraint are associated with length-scale constraints (see comments in Appendix A). of $\mathscr{V}^{*}$ :

Lastly, use is made of the Taylor series expansion of the average field from the centroid

$$
\langle\Psi\rangle^{\mathrm{f}}(\mathbf{r})=\langle\Psi\rangle^{\mathrm{f}}(\mathbf{x})+\mathbf{y} \cdot \nabla\langle\Psi\rangle^{\mathrm{f}}(\mathbf{x})+\frac{1}{2} \mathbf{y y}: \nabla \nabla\langle\Psi\rangle^{\mathrm{f}}(\mathbf{x})+\ldots
$$

where $\mathbf{r}=\mathbf{x}+\mathbf{y}$, yielding

$$
\Psi^{*}(\mathbf{r})=\langle\Psi\rangle^{\mathrm{f}}(\mathbf{x})+\mathbf{y} \cdot \nabla\langle\Psi\rangle^{\mathrm{f}}(\mathbf{x})+\frac{1}{2} \mathbf{y y}: \nabla \nabla\langle\Psi\rangle^{\mathrm{f}}(\mathbf{x})+\ldots+\widetilde{\Psi}^{*}
$$

The following developments require several order of magnitude estimates and comparisons. The complete developments will not be detailed for brevity, as they do not deviate significantly from the analysis performed for upscaling. The resulting length-scale constraints are commented in Appendix A. 


\subsubsection{Flow problem}

Whitaker (1986) proves that

$$
\nabla \cdot\langle\mathbf{v}\rangle^{\mathrm{f}}=-\frac{\nabla \varepsilon}{\varepsilon} \cdot\langle\mathbf{v}\rangle^{\mathrm{f}}
$$

Eq. (23) then allows to write:

$$
\nabla \cdot\langle\mathbf{v}\rangle^{\mathrm{f}}=0
$$

Accounting for this fact and inserting the spatial decomposition into the mass balance equation (1a) yields

$$
\nabla \cdot \widetilde{\mathbf{v}}^{*}=0
$$

By substituting the decomposition (29) in Eq. (1b) and performing order of magnitude estimates, the momentum equation for $\widetilde{\mathbf{v}}^{*}$ becomes

$$
\begin{array}{r}
\rho\left(\nabla \cdot\left(\widetilde{\mathbf{v}}^{*} \widetilde{\mathbf{v}}^{*}\right)+\nabla \cdot\left(\widetilde{\mathbf{v}}^{*}\langle\mathbf{v}\rangle^{\mathrm{f}}(\mathbf{x})\right)+\nabla \cdot\left(\langle\mathbf{v}\rangle^{\mathrm{f}}(\mathbf{x}) \widetilde{\mathbf{v}}^{*}\right)+\left(\langle\mathbf{v}\rangle^{\mathrm{f}}(\mathbf{x})+\widetilde{\mathbf{v}}^{*}\right) \cdot \nabla\langle\mathbf{v}\rangle^{\mathrm{f}}(\mathbf{x})\right) \\
=\mu \nabla^{2} \widetilde{\mathbf{v}}^{*}-\nabla \widetilde{p}^{*}+\mu \nabla^{2}\langle\mathbf{v}\rangle^{\mathrm{f}}(\mathbf{x})-\nabla\langle p\rangle^{\mathrm{f}}(\mathbf{x})
\end{array}
$$

It should be noted that such a development does not require the periodicity of the medium; however, it assumes periodicity of the problem for the deviation fields, as in the case of volume averaging, and this assumption drives the way the cell problem is developed, forcing the elimination of non-periodic terms. An order of magnitude analysis, not detailed here for conciseness, allows to determine the length-scale constraints ensuring the negligibility of the non-periodic terms. The length-scale constraints resulting from the analysis are identical to those implied by the upscaling procedure (see Appendix A for comments on this matter). These length-scale constraints can be summarized by the more conservative Eq. (8).

The processing of the no-slip boundary condition follows similar principles, and we get the complete cell problem for the flow:

$$
\begin{array}{rlrl}
\nabla \cdot \widetilde{\mathbf{v}}^{*} & =0 & & \text { in } \mathscr{V}_{\mathrm{f}}^{*} \\
\rho & {\left[\nabla \cdot\left(\widetilde{\mathbf{v}}^{*} \widetilde{\mathbf{v}}^{*}\right)+\nabla \cdot\left(\widetilde{\mathbf{v}}^{*}\langle\mathbf{v}\rangle^{\mathrm{f}}(\mathbf{x})\right)\right.} & \\
+\nabla \cdot\left(\langle\mathbf{v}\rangle^{\mathrm{f}}(\mathbf{x}) \widetilde{\mathbf{v}}^{*}\right) & & \\
+ & \left.\left(\langle\mathbf{v}\rangle^{\mathrm{f}}(\mathbf{x})+\widetilde{\mathbf{v}}^{*}\right) \cdot \nabla\langle\mathbf{v}\rangle^{\mathrm{f}}(\mathbf{x})\right] & \\
& =\mu \nabla^{2} \widetilde{\mathbf{v}}^{*}-\nabla \widetilde{p}^{*}+\mu \nabla^{2}\langle\mathbf{v}\rangle^{\mathrm{f}}(\mathbf{x})-\nabla\langle p\rangle^{\mathrm{f}}(\mathbf{x}) & & \text { in } \mathscr{V}_{\mathrm{f}}^{*} \\
\widetilde{\mathbf{v}}^{*} & =-\langle\mathbf{v}\rangle^{\mathrm{f}}(\mathbf{x}) & & \text { at } \mathscr{A}^{*} \\
\widetilde{\mathbf{v}}^{*}\left(\mathbf{r}+\mathbf{l}_{i}\right) & =\widetilde{\mathbf{v}}^{*}(\mathbf{r}) & \\
\left\langle\widetilde{\mathbf{v}}^{*}\right\rangle(\mathbf{x}) & =\mathbf{0} & & \\
\widetilde{p}^{*}\left(\mathbf{r}+\mathbf{l}_{i}\right) & =\widetilde{p}^{*}(\mathbf{r}) & \\
\left\langle\widetilde{p}^{*}\right\rangle(\mathbf{x}) & =0 &
\end{array}
$$

The solution of this problem depends on $\langle\mathbf{v}\rangle^{\mathrm{f}}(\mathbf{x}), \nabla\langle\mathbf{v}\rangle^{\mathrm{f}}(\mathbf{x}), \nabla^{2}\langle\mathbf{v}\rangle^{\mathrm{f}}(\mathbf{x})$ and $\nabla\langle p\rangle^{\mathrm{f}}(\mathbf{x})$. Since the macroscopic flow is incompressible, $\nabla\langle p\rangle^{\mathrm{f}}(\mathbf{x})$ and $\langle\mathbf{v}\rangle^{\mathrm{f}}(\mathbf{x})$ are not independent. Therefore, when solving this problem using a penalty method (see Sect. 3.2), only the velocity will be used as a source. The reconstruction of the velocity and pressure fields is done using decomposition (29), where the Taylor series may be truncated at an order ensuring a satisfactory accuracy for the average field. 


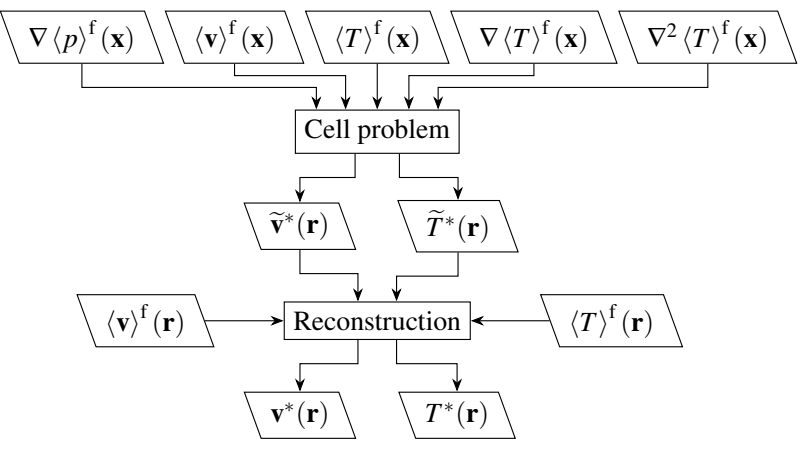

Figure 2: Overview of the dependencies between the fields used in the cell problem and the reconstruction process. For the reconstruction process, $\langle\mathbf{v}\rangle^{\mathrm{f}}(\mathbf{r})$ and $\langle T\rangle^{\mathrm{f}}(\mathbf{r})$ can be approximated by stopping the Taylor series expansion (see Eq. (29)) at a given order.

\subsubsection{Energy problem}

Following the same methodology, we develop the cell problem for energy. For conciseness, no detail is given here. Appendix B contains developments for the boundary condition at $\mathscr{A}^{*}$. The cell problem for the energy equation is:

$$
\begin{aligned}
& \rho c_{p}\left[\nabla \cdot\left(\left(\langle\mathbf{v}\rangle^{\mathrm{f}}(\mathbf{x})+\widetilde{\mathbf{v}}^{*}\right) \widetilde{T}^{*}\right)\right. \\
& \left.+\left(\langle\mathbf{v}\rangle^{\mathrm{f}}(\mathbf{x})+\widetilde{\mathbf{v}}^{*}\right) \cdot \nabla\langle T\rangle^{\mathrm{f}}(\mathbf{x})\right] \\
& =\nabla \cdot\left(k \nabla \widetilde{T}^{*}\right)+k \nabla^{2}\langle T\rangle^{\mathrm{f}}(\mathbf{x}) \quad \text { in } \mathscr{V}_{\mathrm{f}}^{*} \\
& \mathbf{n} \cdot k \nabla \widetilde{T}^{*}=-\mathbf{n} \cdot k \nabla\langle T\rangle^{\mathrm{f}}(\mathbf{x}) \\
& \mathbf{n} \cdot k \nabla \widetilde{T}^{*}=-\mathbf{n} \cdot k \nabla\langle T\rangle^{\mathrm{f}}(\mathbf{x})+S\left(\langle T\rangle^{\mathrm{f}}(\mathbf{x})+\widetilde{T}^{*}\right) \quad \text { at } \mathscr{R}^{*} \\
& \widetilde{T}^{*}\left(\mathbf{r}+\mathbf{l}_{i}\right)=\widetilde{T}^{*}(\mathbf{r}) \\
& \left\langle\widetilde{T}^{*}\right\rangle(\mathbf{x})=0
\end{aligned}
$$

The dependencies of the solution of this problem are those of the flow cell problem (i.e. $\langle\mathbf{v}\rangle^{\mathrm{f}}(\mathbf{x})$ and $\left.\nabla\langle p\rangle^{\mathrm{f}}(\mathbf{x})\right)$, as well as $\langle T\rangle^{\mathrm{f}}(\mathbf{x}), \nabla\langle T\rangle^{\mathrm{f}}(\mathbf{x})$ and $\nabla^{2}\langle T\rangle^{\mathrm{f}}(\mathbf{x})$. Fig. 2 highlights the dependencies between the fields involved in the cell problem and the field reconstruction process.

The derivation of Eqs. (35) required to force periodicity, which a fundamental difference with the reference problem. However, the cell problem retains the nonlinearity of the source term through Eq. (35c) and contain macro-scale information through the macroscopic source terms. Then, the reconstruction process (see Eq. (29)) allows to use additional macro-scale information to build an estimate $T^{*}$ of the $T$ field. It is then $T^{*}$ which is used to compute an estimate of $\langle S\rangle^{\mathscr{R}}$ (see Sect. 4).

It should be noted that in practice, the macroscopic information can originate from either

1. an upscaled macroscopic transport model,

2. a phenomenological macroscopic transport model,

3. or from experimental data. 


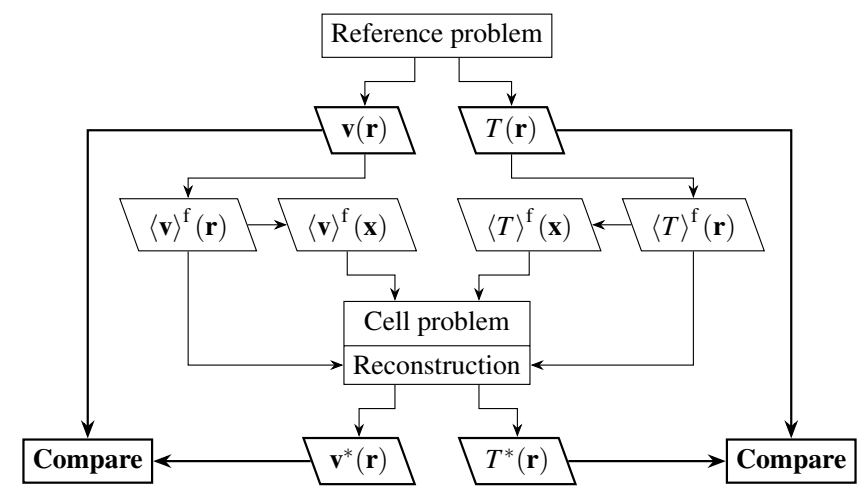

Figure 3: Principle of the validation procedure.

Cases 1 and 2 can apply in the multiscale approach mentioned in Sect. 2.3. Case 3 enables to retrieve pore-scale information from the combination of macro-scale experimental results and knowledge of the pore-scale geometry and physics of the system.

\subsection{Validation}

The validation process of this methodology consists in comparing the results obtained with the downscaled model (Eqs. (34) and (35)) with a reference solution obtained by solving Eqs. (1) and (2). The source terms for the cell problem are computed from the reference solution to ensure consistency between the reference and downscaled solutions. Fig. 3 summarizes the methodology.

\subsubsection{Problem specification}

The chosen reference geometry is an array of $10 \times 7$ cylinders of radius $r_{\mathrm{c}}$ (Fig. 4a). The size of the unit cell is denoted $r_{0}$. The reactive interface covers half of $\mathscr{A}$ and is located in the axial direction of the flow (Fig. 4b). The geometrical and thermophysical quantities involved are arbitrarily chosen for convenience (see Tab. 1).

The boundary conditions are chosen as follows:

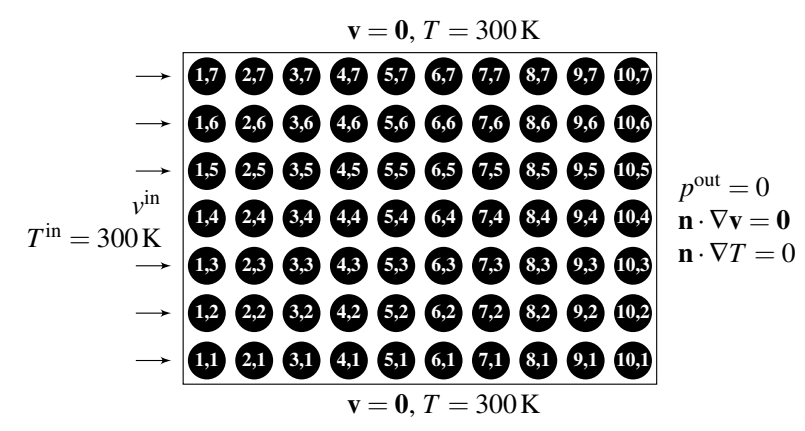

(a)

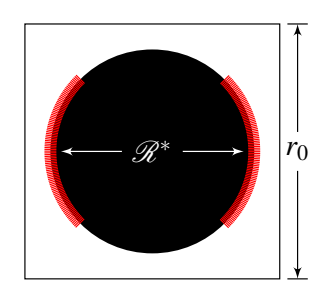

(b)

Figure 4: (a) Reference geometry (the $(m, n)$ couples identify each cell); (b) unit cell. 
- $x=-0.5 r_{0}$ : fixed inlet velocity $v^{\text {in }} \mathbf{e}_{x}$, fixed temperature $T^{\text {in }}$,

- $x=9.5 r_{0}$ : fixed pressure $p^{\text {out }}, \mathbf{e}_{x} \cdot \nabla \mathbf{v}=\mathbf{0}, \mathbf{e}_{x} \cdot \nabla T=0$;

- $y=-0.5 r_{0}$ and $y=6.5 r_{0}: \mathbf{v}=\mathbf{0}, \mathbf{e}_{y} \cdot \nabla T=0$.

The heterogeneous source term expression is

$$
S(T)=f(T) g\left(\mathrm{Pe}^{\mathrm{in}}\right)
$$

with

$$
\begin{aligned}
& f(T)=\left\{\begin{aligned}
10^{-3} T\left(1.333-4.444 \times 10^{-4} T\right) & \text { if } T \in[0 \mathrm{~K}, 3000 \mathrm{~K}] \\
0 & \text { otherwise }
\end{aligned}\right. \\
& g\left(\mathrm{Pe}^{\mathrm{in}}\right)=10\left(1+\mathrm{Pe}^{\mathrm{in}}\right)
\end{aligned}
$$

where $\mathrm{Pe}^{\mathrm{in}}$ is the inlet Péclet number, defined as

$$
\mathrm{Pe}^{\text {in }}=\frac{v^{\text {in }} l}{a} \quad \text { with } l=\frac{1}{A}
$$

Similarly, an inlet Reynolds number $\mathrm{Re}^{\text {in }}$ can be defined. The expression chosen for $S$ is strongly nonlinear and allows coverage of most of the $[0 \mathrm{~K}, 3000 \mathrm{~K}]$ range over the entire geometry for values of $\mathrm{Pe}^{\mathrm{in}}$ ranging from 0.01 to 100 .

\subsubsection{Results}

All computations are made using the OpenFOAM software package (Jasak et al., 2007). The reference problem is solved using the SIMPLE algorithm for the flow part, and a pseudo-time stepping approach for the energy part. The mesh used is unstructured, made of approximately 230000 triangular elements. The covered number range for the inlet Péclet is $[0.01,100]$, the upper limit (which corresponds to $\mathrm{Re}^{\mathrm{in}}=100$ ) being set by the transition to an unsteady flow regime.

Source terms are computed from the reference solution using a second-order filter of the recursively-defined family presented by Angeli et al. (2013) and denoted $m_{2}$ in the following. This ensures continuity of the second-order derivatives of the filtered fields. Filtering yields fields continuously defined over the entire domain $\mathscr{V}$. Since the size of the filter is $3 r_{0}$ (Angeli et al., 2013), the average only makes sense at points distant from $\partial \mathscr{V}$ from at least $1.5 r_{0}$. The filter is applied by considering volume centroids arranged in a $100 \times 70$ grid. The average fields and their derivatives are then evaluated at the centroids of the cells to compute the macroscopic source terms. Average fields inherit the axial symmetry of the reference fields. The average velocity is nearly uniform in the cells not adjacent to $\partial \mathscr{V}$. Fig. 5 shows an example of reference and average temperature fields.

The cell problem is solved for cells $(m, n) \in \llbracket 2,9 \rrbracket \times \llbracket 4,6 \rrbracket$ (see Fig. 4) using the penalty method of Angeli et al. (2013). The momentum problem is solved using a modified SIMPLE algorithm. Relaxation coefficients have to be adjusted with the Reynolds number to ensure numerical stability. The energy problem is solved using a pseudo-time stepping approach. The mesh used has the same element density as that used to solve the reference problem (i.e. about 3300 cells). The Taylor series used for the reconstruction of the velocity and temperature fields is truncated at the first order. 
(a)

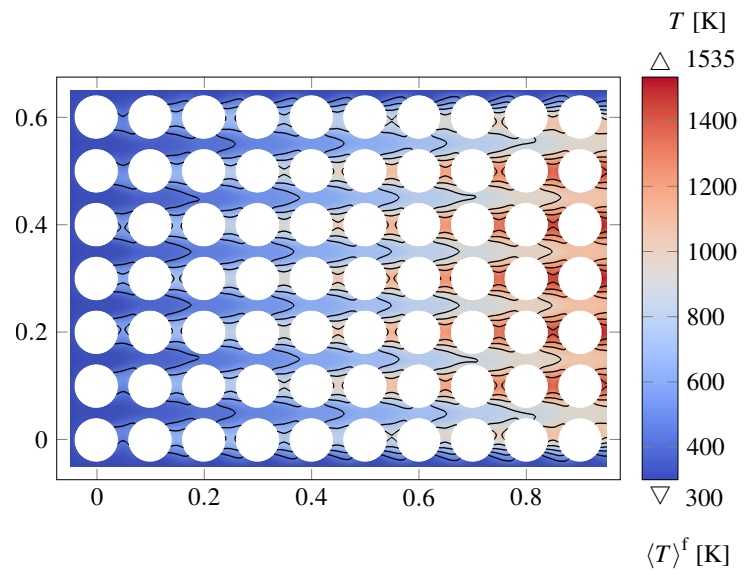

(b)

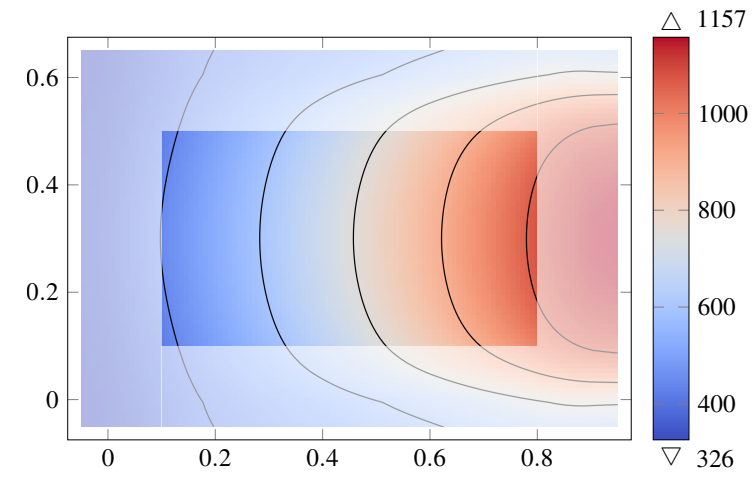

Figure 5: Example of (a) reference and (b) average temperature fields $\left(\mathrm{Pe}^{\mathrm{in}}=10\right)$. Maximum and minimum field values are specified. Filtered data is unusable in the faded zone due to the effective size of the recursive filter. 

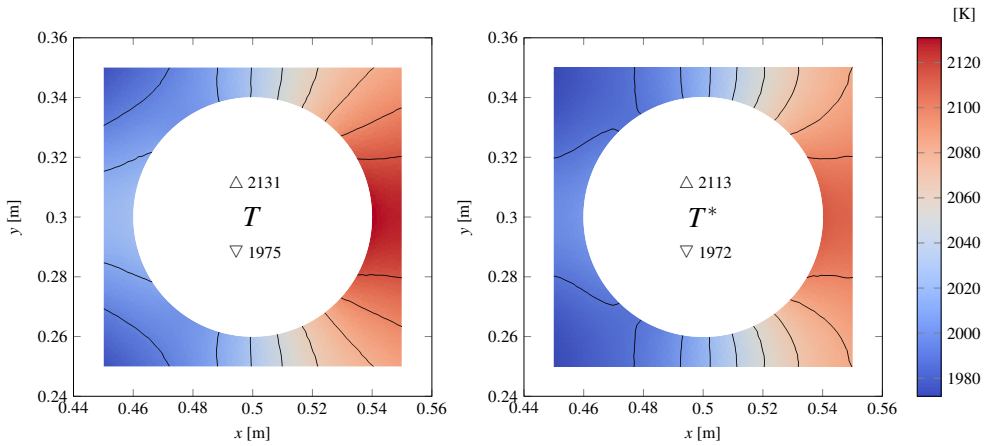

(b)
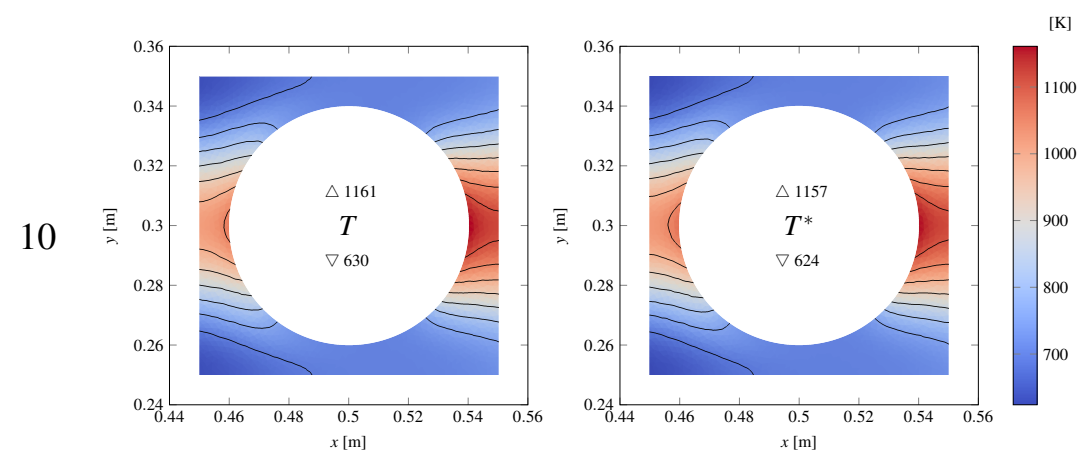

Figure 6: Reference and reconstructed temperature fields in cell $(6,4)$ for (a) $\mathrm{Pe}^{\mathrm{in}}=0.1$ and (b) $\mathrm{Pe}^{\text {in }}=10$. Maximum and minimum field values are specified.

Qualitative agreement is good for cells far from the system boundaries $\partial \mathscr{V}$ (Fig. 6): the minimum and maximum values are correctly reproduced. Fig. 6a displays distorted iso- $T^{*}$ lines. This is possibly due to the first-order truncation of the Taylor series. Cell $(6,4)$ is located on the system symmetry line, where $\frac{\mathrm{d}\langle T\rangle^{\mathrm{f}}}{\mathrm{dy}}=0$ : the shape of the $\langle T\rangle^{\mathrm{f}}$ profile in the $y=$ constant plane is therefore dominated by the second-order term of the Taylor series in this cell. Since Fig. 6a corresponds to a low Péclet regime, the local temperature profile is dominated by the variations of $\langle T\rangle^{\mathrm{f}}$, thus explaining the discrepancies. On the contrary, in the high Péclet number regime (Fig. 6b), the local temperature profile is dominated by deviations from the average, ensuring an excellent accuracy.

Cells closer to $\partial \mathscr{V}$ display higher errors and physically irrelevant behavior due to the irrelevance of periodic boundary conditions in such areas (Fig. 7). This is consistent with the results of Angeli et al. (2013). Fig. 8 displays the temperature at $\mathscr{A}^{*}$ and supports these conclusions.

We define the average relative error at $\mathscr{A}^{*}$ as

$$
\mathscr{S}_{2}=\sqrt{\frac{1}{\mathscr{A}^{*}} \int_{\mathscr{A}^{*}}\left(\frac{T^{*}-T}{T}\right)^{2} \mathrm{~d} \mathscr{A}}
$$

This indicator remains below $2 \%$ in all cells for $\mathrm{Pe}^{\text {in }} \leq 1$ (Fig. 9a to c), which proves the relevance of downscaling for surface temperature reconstruction. For higher values of the Péclet number (Fig. 9d and e), the accuracy is significantly degraded in near-entry cells; however, the accuracy remains good for cells located far from the inflow boundary. Accuracy 

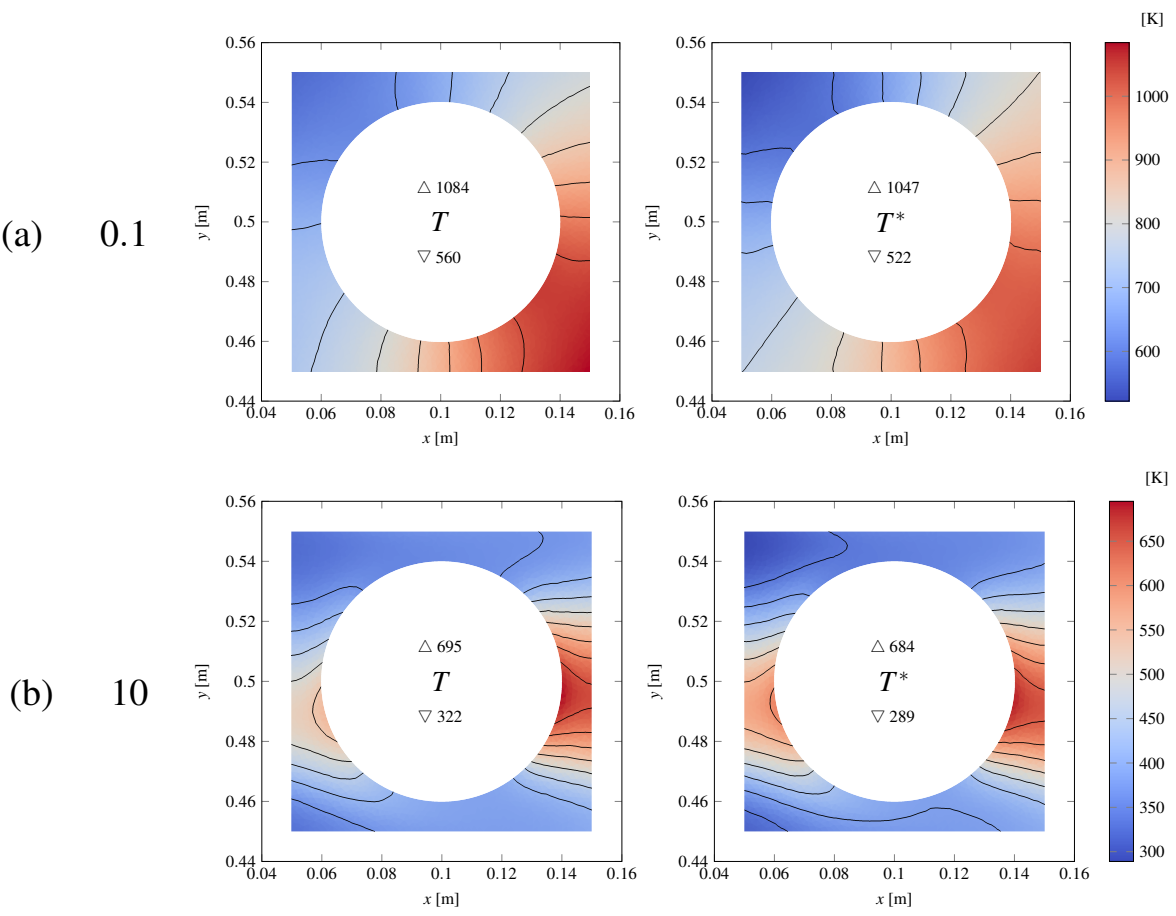

Figure 7: Reference and reconstructed temperature fields in cell $(2,6)$ for (a) $\mathrm{Pe}^{\mathrm{in}}=0.1$ and (b) $\mathrm{Pe}^{\text {in }}=10$. Maximum and minimum field values are specified. 
$\mathrm{Pe}^{\mathrm{in}}$

$(6,4)$

(a)

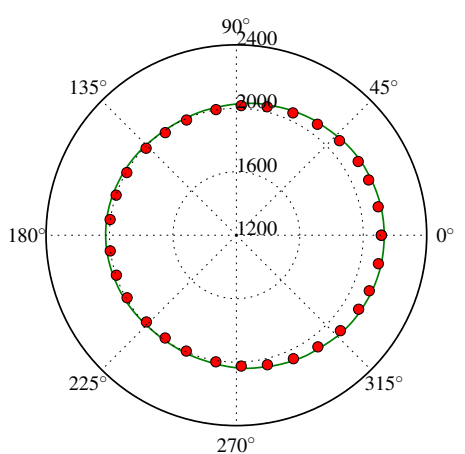

(b)

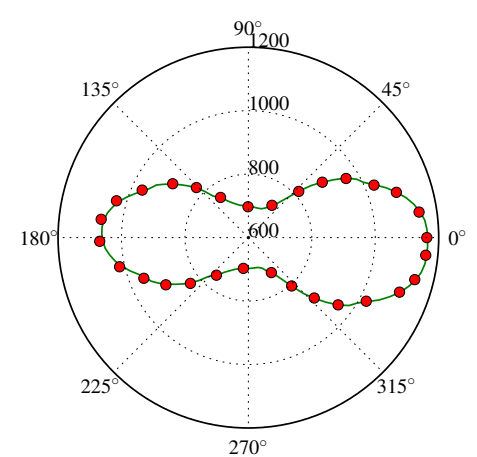

$(2,6)$
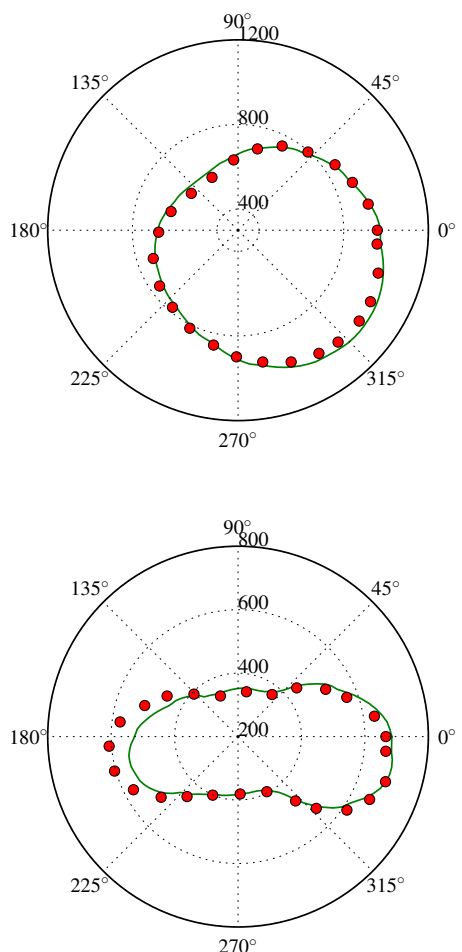

Figure 8: Reference $(-T)$ and reconstructed $\left(\bigcirc T^{*}\right)$ temperature at the interface $\mathscr{A}^{*}$ for (a) $\mathrm{Pe}^{\text {in }}=0.1$ and $(\mathrm{b}) \mathrm{Pe}^{\mathrm{in}}=10$ in cells (left) $(6,4)$ and (right) $(2,6)$. 
is expectedly lower when pore-scale variations of $T^{*}$ increase (see Eq. (55)). It should be noted that since some phenomena involve high temperature sensitivity in specific ranges (e.g. phase change), the required accuracy might be lower than $1 \%$ in practical cases.

\section{Macroscopic model correction}

In the conditions where the downscaling methodology is relevant, the accuracy of the solution yielded by the downscaling procedure is sufficient to allow the computation of a good estimate of a macroscopic source term. In this section, we present a multiscale approach in which downscaling is successfully used to compute a nonlinear source term.

A reference problem is described and solved. The resulting solution is averaged and compared with two macroscopic models, one using a straightforward expression for the macro-scale source term, and another one taking advantage of downscaling to apply a multiscale correction to the source term.

\subsection{Reference problem}

The considered reference geometry is a 20-column array of cylinders (Fig. 10). The governing equations are Eqs. (1) and (2). Boundary conditions are:

- $x=-0.5 r_{0}$ : fixed inlet velocity $v^{\text {in }} \mathbf{e}_{x}$, fixed temperature $T^{\text {in }}$;

- $x=19.5 r_{0}$ : fixed pressure $p^{\text {out }}, \mathbf{e}_{x} \cdot \nabla \mathbf{v}=\mathbf{0}, \mathbf{e}_{x} \cdot \nabla T=0$;

- $y=-0.5 r_{0}$ and $y=0.5 r_{0}$ : periodic boundary conditions.

The expression of the heterogeneous source term is given by Eq. (36) with

$$
\begin{aligned}
f(T) & =\left\{\begin{array}{cl}
10^{-3} T\left(1.333-4.444 \times 10^{-4} T\right) & \text { if } T \in[0 \mathrm{~K}, 3000 \mathrm{~K}] \\
0 & \text { otherwise }
\end{array}\right. \\
g\left(\mathrm{Pe}^{\text {in }}\right) & =7.5\left(0.01+\mathrm{Pe}^{\text {in }}\right)
\end{aligned}
$$

with $\mathrm{Pe}^{\text {in }}$ defined by Eq. (37). Values for geometrical quantities are taken from Tab. 2 while thermophysical properties are taken from Tab. 1 . The reference problem is solved for $\mathrm{Pe}^{\mathrm{in}} \in\{0.1,1,10\}$. An average reference solution field $\langle T\rangle_{\text {ref }}^{\mathrm{f}}$ is obtained for $x \in\left[r_{0}, 18 r_{0}\right]$ by applying the $m_{2}$ filter used in Sect. 3.2.

Since the flow is incompressible, the average velocity $\langle\mathbf{v}\rangle_{\text {ref }}^{\mathrm{f}}$ is uniform in the range $x \in\left[r_{0}, 18 r_{0}\right]$. Its value will be used as the macroscopic velocity.

\subsection{Macroscopic problem}

The macroscopic geometry is one-dimensional, corresponding to the $x \in\left[r_{0}, 18 r_{0}\right]$ range in the reference geometry (Fig. 10). The flow being incompressible, the macroscopic velocity has consequently a uniform value $\overline{\mathbf{v}}$, obtained from the reference solution:

$$
\overline{\mathbf{v}}=\langle\mathbf{v}\rangle_{\text {ref }}^{\mathrm{f}}\left(x=10 r_{0}\right)=\bar{v} \mathbf{e}_{x}
$$

It is therefore unnecessary to solve a macroscopic flow equation: $\bar{v}$ can be imposed. 
$\mathrm{Pe}^{\text {in }}$

(a)

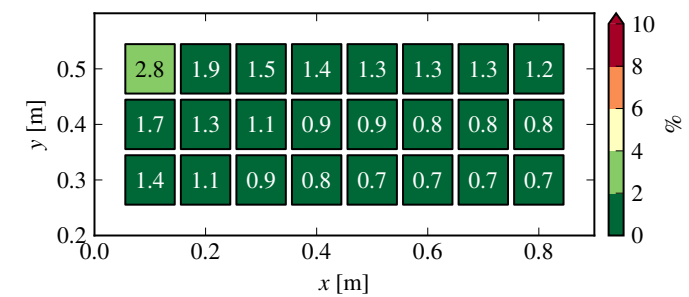

(b)

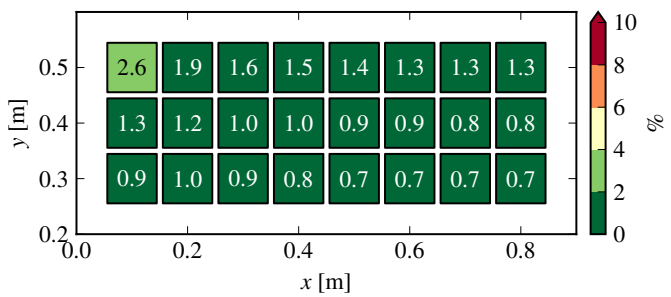

(c) 1

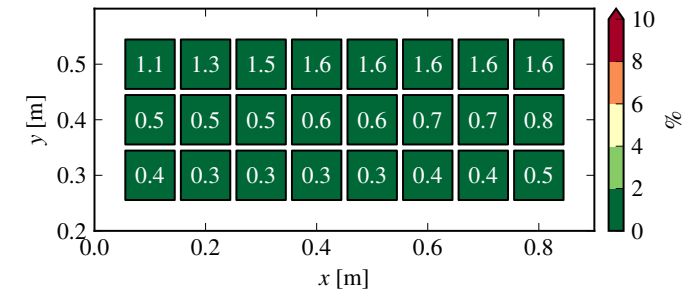

(d) 10
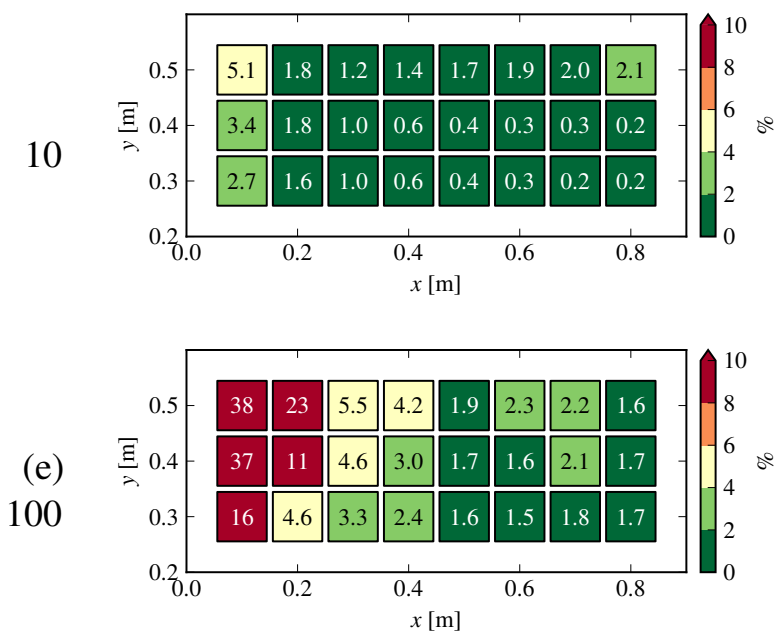

Figure 9: Average relative error $\mathscr{S}_{2}$ (see Eq. (38)) for $\mathrm{Pe}^{\mathrm{in}}=$ (a) 0.01, (b) 0.1, (c) 1, (d) 10 and (e) 100 . Only cells above or on the symmetry line are represented. 


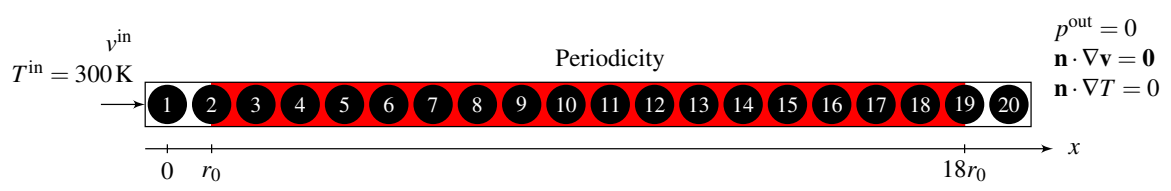

Figure 10: Reference geometry and macroscopic simulation domain (in red).

The energy balance is heuristically modeled by using Eq. (18), setting $\alpha=0$ and neglecting the last term. This yields the following energy equation:

$$
\rho c_{p} \varepsilon \bar{v} \frac{\mathrm{d} \bar{T}}{\mathrm{~d} x}=K_{x x} \frac{\mathrm{d}^{2} \bar{T}}{\mathrm{~d} x^{2}}+R \bar{S}
$$

where $K_{x x}$ is the $x x$ component of the effective conductivity tensor and $\bar{S}$ is an approximate of $\langle S\rangle^{\mathscr{R}}$ to be defined in the following. $K_{x x}$ is obtained from the work of Quintard et al. (1997) (see Appendix C).

To minimize potential inaccuracy, boundary conditions are obtained from the reference solution:

$$
\begin{gathered}
\bar{T}\left(x=r_{0}\right)=\langle T\rangle_{\text {ref }}^{\mathrm{f}}\left(x=r_{0}\right) \\
\frac{\mathrm{d} \bar{T}}{\mathrm{~d} x}\left(x=18 r_{0}\right)=\frac{\mathrm{d}\langle T\rangle_{\text {ref }}^{\mathrm{f}}}{\mathrm{d} x}\left(x=18 r_{0}\right)
\end{gathered}
$$

\subsection{Principle of multiscale source term correction}

As previously mentioned, the macroscopic heat transfer model is obtained by combining conclusions from upscaling using the volume averaging method and a heuristic approach. In Eq. (40a), the way $\bar{S}$ is evaluated still remains unspecified, because, as mentioned in Sect. 2, the volume averaging method applied to a heat transfer problem in the presence of a nonlinear heterogeneous source term does not yield an expression for the macroscopic source term $\langle S\rangle^{\mathscr{R}}$ as a function of macroscopic variables.

Sect. 3 showed that downscaling yields a cell problem whose solution is a function of macro-scale variables and features reasonable accuracy in a variety of cases. Therefore, it is possible to get an approximate of $\langle S\rangle^{\mathscr{R}}$ defined as

$$
\langle S\rangle^{\mathscr{R}} \simeq \frac{1}{\mathscr{R}^{*}} \int_{\mathscr{R}^{*}} S\left(T^{*}\right) \mathrm{d} \mathscr{A}
$$

A multiscale approach (see Fig. 11a) can therefore be used to solve a macroscopic-scale problem:

1. Solve problem (40) a first time using a zeroth-order Taylor series truncation to compute the source term (see Eq. (22)):

$$
\bar{S}=\bar{S}_{0}=S(\bar{T})
$$

This solution will be referred to as the baseline solution.

2. Use $\bar{T}$ to generate source terms for cell problems at a set $\left\{\mathbf{x}_{i}\right\}_{i \in \llbracket 2,19 \rrbracket}$ of locations, where $\mathbf{x}_{i}$ is the centroid of cell $i$ (see Fig. 10). 
(a)

Baseline
Correction

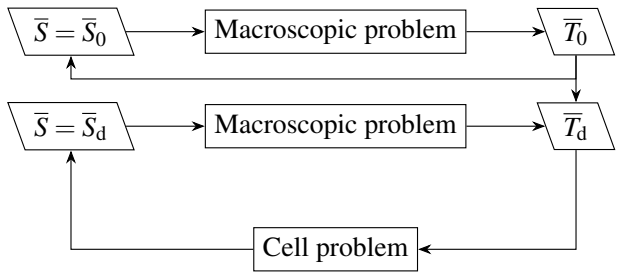

(b)

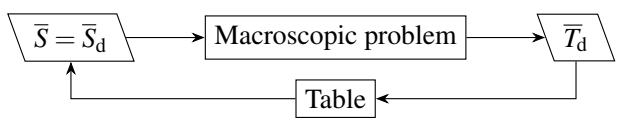

Figure 11: Principle of the source term correction with (a) a multiscale approach and (b) a table-based approach. The baseline iteration loop is formal and is not necessarily implemented using an iterative algorithm.

3. Solve the cell problems to retrieve a downscaling-based estimate for $\bar{S}$ :

$$
\bar{S}\left(\mathbf{x}_{i}\right)=\bar{S}_{\mathrm{d}}\left(\mathbf{x}_{i}\right)=\frac{1}{\mathscr{R}^{*}} \int_{\mathscr{R}^{*}} S\left(T^{*}\right) \mathrm{d} \mathscr{A}
$$

The values for the $\bar{S}$ field outside the $\left\{\mathbf{x}_{i}\right\}$ set are obtained by interpolation.

4. Use the corrected $\bar{S}$ field to recompute the solution of problem (40).

5. Repeat steps 2 to 5 until convergence is achieved.

\subsection{Source term tabulation}

While the multiscale approach for source term correction is conceptually interesting, implementing it on a large scale may be problematic due to the high computational cost of the correction loop. As mentioned in Sect. 3.1, the dependencies of the cell problem are limited. It is therefore possible to replace the correction loop with a table for $\bar{S}_{\mathrm{d}}$, whose dimensionality is determined by the dependencies of the solution of the cell problem (see Sect. 3 and Fig. 2). This then removes the need to compute the baseline solution (see Fig. 11b).

The reference solution allows the definition of the range of macroscopic dependencies that should be covered by the $\bar{S}_{\mathrm{d}}$ table. Since $\overline{\mathbf{v}}$ is uniform, the flow part of the cell problem only depends on $\overline{\mathbf{v}}$, i.e. on the Péclet number. The downscaled source term therefore depends on $\mathrm{Pe}^{\text {in }}, \bar{T}, \frac{\mathrm{d} \bar{T}}{\mathrm{~d} x}$ and $\frac{\mathrm{d}^{2} \bar{T}}{\mathrm{~d} x^{2}}$.

In the case considered, the variability of $\bar{S}_{\mathrm{d}}$ remains below $0.01 \%$ for $\frac{\mathrm{d}^{2} \bar{T}}{\mathrm{~d} x^{2}} \in\left[0 \mathrm{~K} \mathrm{~m}^{-2}, 1000 \mathrm{Km}^{-2}\right]$ over the entire covered range for $\bar{T}([300 \mathrm{~K}, 900 \mathrm{~K}])$ and $\frac{\mathrm{d} \bar{T}}{\mathrm{~d} x}\left(\left[100 \mathrm{Km}^{-1}, 300 \mathrm{Km}^{-1}\right]\right)$. Therefore, only the dependency versus $\frac{\mathrm{d} \bar{T}}{\mathrm{~d} x}$ and $\bar{T}$ will be considered in the final table (Fig. 12). The dependency versus $\frac{\mathrm{d} \bar{T}}{\mathrm{~d} x}$ is non-negligible only for $\mathrm{Pe}^{\mathrm{in}}=10$, where variability reaches $10 \%$ over the covered range.

\subsection{Results}

Fig. 13 compares the macroscopic temperature profiles obtained using $\bar{S}_{0}$ and $\bar{S}_{\mathrm{d}}$ with the reference solution. At $\mathrm{Pe}^{\mathrm{in}}=0.1$, using $\bar{S}_{\mathrm{d}}$ does not result in an accuracy improvement over 
$P e^{\text {in }}$

(a)

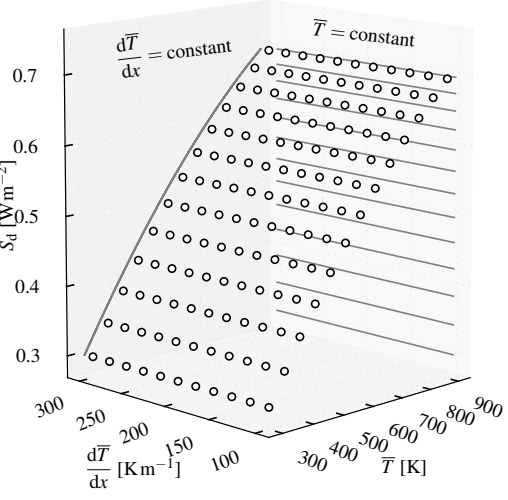

(b) 1

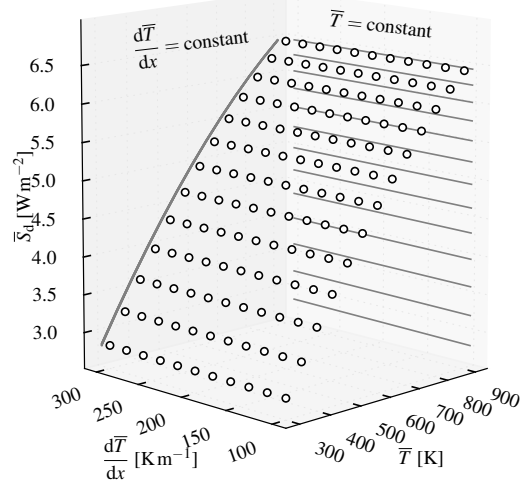

(c) 10

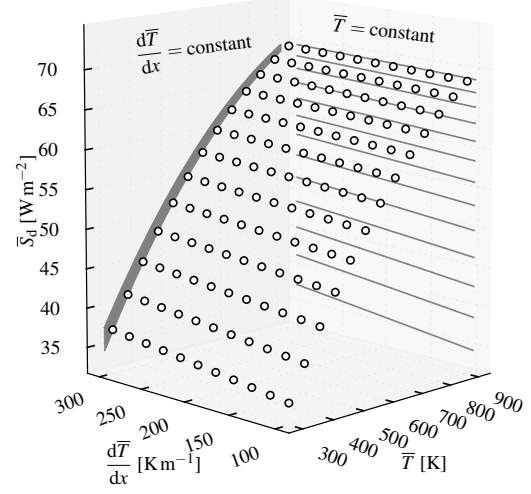

Figure 12: $\bar{S}_{\mathrm{d}}$ versus $\bar{T}$ and $\frac{\mathrm{d} \bar{T}}{\mathrm{~d} x}$ for $\mathrm{Pe}^{\mathrm{in}}=$ (a) 0.1 , (b) 1 and (c) 10 (with projections for constant $\bar{T}$ and $\frac{\mathrm{d} \bar{T}}{\mathrm{~d} x}$ ). 

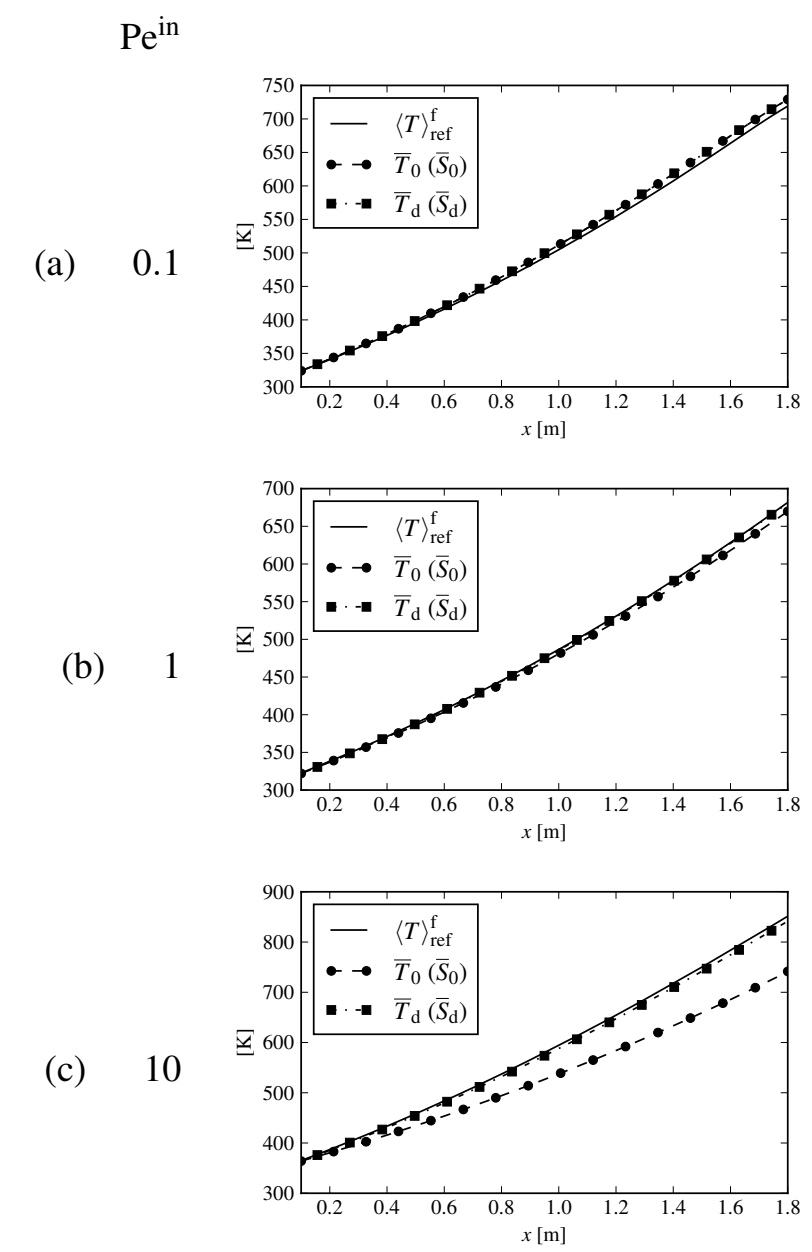

Figure 13: $\bar{T}$ compared to $\langle T\rangle_{\text {ref }}^{\mathrm{f}}$ using $\bar{S}_{0}$ and $\bar{S}_{\mathrm{d}}$ for $\mathrm{Pe}^{\mathrm{in}}=$ (a) 0.1 , (b) 1 and (c) 10 . 
$\bar{S}_{0}$. This is due to the uniformity of the temperature field at the pore scale: in such diffusive regime, the temperature deviation field has low values compared to the average, resulting in an interface temperature close to the average temperature in the cell. The residual error on the temperature profile might be due to the strong influence of boundary conditions, or to the approximations made to build Eq. (40a) from the volume-averaged formulation.

At $\mathrm{Pe}^{\mathrm{in}}=1$, where conduction and convection have comparable influences, the change of value for $\bar{S}$ is noticeable, although the variation in the outlet temperature is lower than the error visible for $\mathrm{Pe}^{\text {in }}=0.1$.

At $\mathrm{Pe}^{\mathrm{in}}=10$, where convection is the dominating transfer mode, $\bar{S}_{\mathrm{d}}$ yields significantly better results than $\bar{S}_{0}$. It is consistent with the fact that, in this regime, the temperature at $\mathscr{R}$ noticeably deviates from the average temperature: therefore, the heterogeneous source term is much better estimated by $\bar{S}_{\mathrm{d}}$ than $\bar{S}_{0}$.

\section{Conclusion}

The challenges brought by nonlinearity of heterogeneous source terms when performing upscaling using the volume averaging method were highlighted with a simple case. Estimating the fluid-solid temperature is critical, even when using a simple model with a reduced number of dependencies. Use of a downscaling methodology allows to estimate the temperature field in the averaging volume, provided that proper length-scale constraints are satisfied.

The downscaling methodology developed by Angeli et al. (2013) was extended to include nonlinear heterogeneous source terms and the results were validated against the reference temperature field for an inlet Péclet number ranging from 0.01 to 100 . The accuracy of the reconstructed interface temperature field is excellent in the bulk of the medium for every Péclet number, and it is expectedly questionable near the medium boundaries.

The results of the downscaling procedure were used to tabulate the effective macroscopic heterogeneous source term against the inlet Péclet number, the macroscopic temperature and its gradient. Use of this estimate significantly improves the results of a macroscopic model compared to the simple uniform temperature approximation when the interface temperature significantly deviates from the fluid phase average temperature. This approach represents a good compromise between accuracy and accounting for multiscale effects. In the presence of steep macroscopic physical field variations, however, specific approaches have to be used, e.g. direct modeling of the front (Debenest et al., 2005). It should be noted that downscaling can be used in such cases to provide boundary conditions (Angeli, 2011).

For the purpose of macro-scale source term tabulation, a downscaling analysis has the advantage of allowing an unambiguous determination of the dependencies of the resulting macroscopic source term. While this approach was developed for a physically unrealistic case for simplicity, it can be readily extended to more general heat (Quintard and Whitaker, 2000; Quintard et al., 2000; Leroy et al., 2013) and mass (Guo et al., 2015) transfer problems. Downscaling also provides a way to determine the $\alpha$ mapping coefficient in closure problem II (see Appendix C), therefore removing the need for an iterative resolution procedure of closure problems. 


\section{A Comments on length-scale constraints}

This study, being based on the volume averaging method, requires the analysis of terms in local equations at multiple stages of development. Terms in equations are estimated and compared with the others to decide on which ones are dominant. The estimation of terms is based on the analysis of their order of magnitude. This is a very common practice in the volume averaging literature.

It is important to firstly distinguish the purpose of estimates. When performing upscaling with the volume averaging method for macroscopically uniform media, simplifications are done at both the pore scale and the macroscopic scale:

- at the macroscopic scale, it is important to ensure that insignificant terms are not kept, as they would introduce unnecessary complexity;

- at the pore scale, the goal is to ensure that all the fields involved are either constant or periodic over the averaging volume (or can be forcibly considered so), in order to set ground for the usage of periodic boundary conditions.

The principles of this analysis are presented by Whitaker (1999), and thorough details are given by Quintard and Whitaker (1994a,b,c,d,e).

The length scale analysis required to perform the upscaling of the problem presented in Sect. 2 is performed in the more general case presented by Whitaker (1998) and can be summarized by:

- the deviation equation can be written in a local form if

$$
\begin{aligned}
\frac{l_{T_{0}}}{r_{0}} & \ll 1 \\
\frac{r_{0}^{2}}{L_{\varepsilon} L_{T, 1}} & \ll 1
\end{aligned}
$$

- the average of deviations can be neglected and the average of a quantity can be considered constant over the averaging volume if

$$
\begin{aligned}
\frac{l_{T_{0}}}{L_{T, 0}} & \ll 1 \\
\frac{r_{0}^{2}}{L_{T, 0} L_{T, 1}} & \ll 1
\end{aligned}
$$

In Eqs. (44), $l_{T, 0}$ is the typical variation length scale of $T, r_{0}$ is the size of the averaging volume, $L_{\varepsilon}$ is the typical variation length scale of porosity and $L_{T, n}$ is the typical variation length scale for the $n$-th derivative of $\langle T\rangle^{\mathrm{f}}$ (e.g. $L_{T, 1}$ is the typical variation length scale of $\left.\nabla\langle T\rangle^{\mathrm{f}}\right)$. It is worth mentioning that to our knowledge, terms with an order higher than 2 in the Taylor series developments used for length scale analysis is always omitted; repeating the analysis for orders higher than 2 yields constraints of the form of Eq. (54). In the case considered, to the classic set of constraints (44) must be added constraint (55), which ensures that a local approximation of the source term is appropriate.

The derivation process for the downscaled equations presented in Sect. (3.1) requires calculus close to that performed for the upscaling procedure, also requiring to provide a 
local form for the cell problem (i.e. approximating the macro-scale varying quantities with constant values). This leads to length-scale constraints identical to those appearing during the volume averaging process. It follows that the length scale constraints of both methodologies are compatible: that using the downscaling methodology is relevant in the same conditions as using the upscaling methodology.

\section{B Development of the flux boundary condition for the energy cell problem}

This appendix contains the developments leading to Eqs. (35b) and (35c). We first insert decomposition (24) into Eq. (2c) and get:

$$
\mathbf{n} \cdot k \nabla \widetilde{T}^{*}=-\mathbf{n} \cdot k \nabla\left(\langle T\rangle^{\mathrm{f}}(\mathbf{x})+\mathbf{y} \cdot \nabla\langle T\rangle^{\mathrm{f}}(\mathbf{x})+\frac{1}{2} \mathbf{y y}: \nabla \nabla\langle T\rangle^{\mathrm{f}}(\mathbf{x})+\ldots\right) \quad \text { at } \mathscr{A}^{*} \backslash \mathscr{R}^{*}
$$

where $\mathbf{r}=\mathbf{x}+\mathbf{y}$.

$\mathbf{n} \cdot k \nabla\left(\langle T\rangle^{\mathrm{f}}(\mathbf{x})\right)$ is equal to zero, and we trivially show that

$$
\mathbf{n} \cdot k \nabla\left(\mathbf{y} \cdot \nabla\langle T\rangle^{\mathrm{f}}(\mathbf{x})\right)=\mathbf{n} \cdot k \nabla\langle T\rangle^{\mathrm{f}}(\mathbf{x})
$$

The remaining terms are non-periodic and can be neglected if

$$
\forall n \in \llbracket 1,+\infty \llbracket, \frac{r_{0}}{L_{T, n}} \ll 1
$$

where $L_{T, n}$ is the typical variation length scale of the $n$-th order derivative of $\langle T\rangle^{\mathrm{f}}$ (e.g. $L_{T, 1}$ is the typical variation length scale of $\nabla\langle T\rangle^{\mathrm{f}}$ ). We therefore write:

$$
\mathbf{n} \cdot k \nabla \widetilde{T}^{*}=-\mathbf{n} \cdot k \nabla\langle T\rangle^{\mathrm{f}}(\mathbf{x}) \quad \text { at } \mathscr{A}^{*} \backslash \mathscr{R}^{*}
$$

Following a similar process, we insert decomposition (24) into Eq. (2b):

$$
\begin{aligned}
\mathbf{n} \cdot k \nabla \widetilde{T}^{*}= & -\mathbf{n} \cdot k \nabla\left(\langle T\rangle^{\mathrm{f}}(\mathbf{x})+\mathbf{y} \cdot \nabla\langle T\rangle^{\mathrm{f}}(\mathbf{x})+\frac{1}{2} \mathbf{y y}: \nabla \nabla\langle T\rangle^{\mathrm{f}}(\mathbf{x})+\ldots\right) \\
& +S\left(\langle T\rangle^{\mathrm{f}}(\mathbf{x})+\mathbf{y} \cdot \nabla\langle T\rangle^{\mathrm{f}}(\mathbf{x})+\frac{1}{2} \mathbf{y y}: \nabla \nabla\langle T\rangle^{\mathrm{f}}(\mathbf{x})+\ldots+\widetilde{T}^{*}\right) \quad \text { at } \mathscr{R}^{*}
\end{aligned}
$$

The gradient term is expanded and processed as before. To process the source term, we define the non-periodic part of $T^{*}$

$$
\begin{aligned}
\Theta^{*} & =T^{*}-\left(\langle T\rangle^{\mathrm{f}}(\mathbf{x})+\widetilde{T}^{*}\right) \\
& =\mathbf{y} \cdot \nabla\langle T\rangle^{\mathrm{f}}(\mathbf{x})+\frac{1}{2} \mathbf{y y}: \nabla \nabla\langle T\rangle^{\mathrm{f}}(\mathbf{x})+\ldots
\end{aligned}
$$

The heterogeneous source term has a non-periodic part due to the presence of $\Theta^{*}$. We will establish the negligibility condition for the non-periodic terms. We first use a Taylor series expansion to write

$$
S\left(T^{*}\right)=\underbrace{S\left(\langle T\rangle^{\mathrm{f}}(\mathbf{x})+\widetilde{T}^{*}\right)}_{\text {periodic }}+\underbrace{\Theta^{*} \frac{\mathrm{d} S}{\mathrm{~d} T}\left(\langle T\rangle^{\mathrm{f}}(\mathbf{x})+\widetilde{T}^{*}\right)+\frac{\Theta^{* 2}}{2} \frac{\mathrm{d}^{2} S}{\mathrm{~d} T^{2}}\left(\langle T\rangle^{\mathrm{f}}(\mathbf{x})+\widetilde{T}^{*}\right)+\ldots}_{\text {non-periodic }}
$$


The first term in the non-periodic part can be expanded:

$$
\Theta^{*} \frac{\mathrm{d} S}{\mathrm{~d} T}\left(\langle T\rangle^{\mathrm{f}}(\mathbf{x})+\widetilde{T}^{*}\right)=\left(\mathbf{y} \cdot \nabla\langle T\rangle^{\mathrm{f}}(\mathbf{x})+\frac{1}{2} \mathbf{y y}: \nabla \nabla\langle T\rangle^{\mathrm{f}}(\mathbf{x})+\ldots\right) \frac{\mathrm{d} S}{\mathrm{~d} T}\left(\langle T\rangle^{\mathrm{f}}(\mathbf{x})+\widetilde{T}^{*}\right)
$$

The first term is estimated

$$
\mathbf{y} \cdot \nabla\langle T\rangle^{\mathrm{f}}(\mathbf{x}) \frac{\mathrm{d} S}{\mathrm{~d} T}\left(\langle T\rangle^{\mathrm{f}}(\mathbf{x})+\widetilde{T}^{*}\right)=\mathscr{O}\left(\frac{r_{0}\langle T\rangle^{\mathrm{f}}(\mathbf{x})}{L_{T, 0}} \frac{\mathrm{d} S}{\mathrm{~d} T}\left(\langle T\rangle^{\mathrm{f}}(\mathbf{x})+\widetilde{T}^{*}\right)\right)
$$

and can be neglected compared to the periodic part if the following constraint is valid:

$$
\frac{r_{0}}{L_{T, 0}}\langle T\rangle^{\mathrm{f}}(\mathbf{x}) \frac{\mathrm{d} S}{\mathrm{~d} T}\left(\langle T\rangle^{\mathrm{f}}(\mathbf{x})+\widetilde{T}^{*}\right) \ll S\left(\langle T\rangle^{\mathrm{f}}(\mathbf{x})+\widetilde{T}^{*}\right)
$$

Similar estimates can be calculated for all terms of Eq. (51), leading to the following constraints:

$$
\forall n \in \llbracket 1,+\infty \llbracket, \frac{r_{0}{ }^{n}}{\prod_{k=0}^{n-1} L_{T, k}}\langle T\rangle^{\mathrm{f}}(\mathbf{x}) \frac{\frac{\mathrm{d} S}{\mathrm{~d} T}\left(\langle T\rangle^{\mathrm{f}}(\mathbf{x})+\widetilde{T}^{*}\right)}{S\left(\langle T\rangle^{\mathrm{f}}(\mathbf{x})+\widetilde{T}^{*}\right)} \ll 1
$$

Finally, the heterogeneous source boundary condition becomes:

$$
\mathbf{n} \cdot k \nabla \widetilde{T}=-\mathbf{n} \cdot k \nabla\langle T\rangle^{\mathrm{f}}(\mathbf{x})+S\left(\langle T\rangle^{\mathrm{f}}(\mathbf{x})+\widetilde{T}^{*}\right) \quad \text { at } \mathscr{R}^{*}
$$

Appendix A contains comments on length scale constraints.

\section{Closure problems for the volume averaging procedure}

The closure problems are derived by inserting Eq. (17) into Eq. (16). This results in two independent closure problems whose solutions depend on the cell Péclet number. The formulations below include the substitution of boundary conditions in the integral terms and take into account the medium homogeneity hypothesis.

Problem I

$$
\begin{aligned}
\rho c_{p} \widetilde{\mathbf{v}} \cdot \nabla \mathbf{b}+\rho c_{p} \mathbf{v} & =\nabla \cdot(k \nabla \mathbf{b}) & & \text { in } \mathscr{V}_{\mathrm{f}}^{*} \\
\mathbf{n} \cdot k \nabla \mathbf{b} & =-\mathbf{n} k & & \text { at } \mathscr{A}^{*} \\
\mathbf{b}\left(\mathbf{r}+\mathbf{l}_{i}\right) & =\mathbf{b}(\mathbf{r}) & & \\
\langle\mathbf{b}\rangle & =\mathbf{0} & &
\end{aligned}
$$

\section{Problem II}

$$
\begin{aligned}
\rho c_{p} \widetilde{\mathbf{v}} \cdot \nabla r & =\nabla \cdot(k \nabla r)-\frac{R^{*}}{\varepsilon} \\
\mathbf{n} \cdot k \nabla r & =1+\alpha \\
\mathbf{n} \cdot k \nabla r & =0 \\
r\left(\mathbf{r}+\mathbf{l}_{i}\right) & =r(\mathbf{r}) \\
\langle r\rangle & =0
\end{aligned}
$$$$
\text { in } \mathscr{V}_{\mathrm{f}}^{*}
$$$$
\text { at } \mathscr{R}^{*}
$$$$
\text { at } \mathscr{A}^{*} \backslash \mathscr{R}^{*}
$$ 


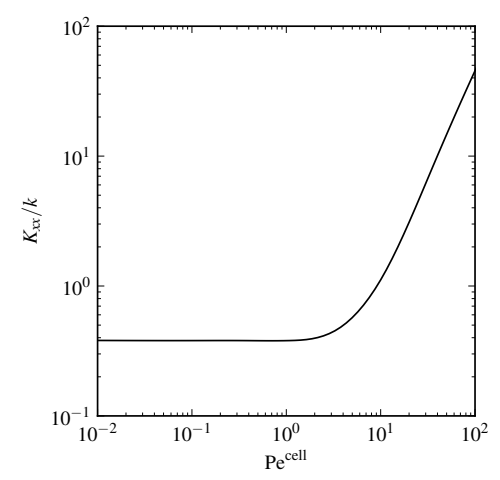

Figure 14: Effective conductivity used in Sect. 4 (versus $\mathrm{Pe}^{\text {cell }}=\bar{v} /(a A)$ ).

Solving the closure problems is beyond the scope of this study. However, the computations performed in Sect. 4 require values for the $K_{x x}$ conductivity tensor coefficient. Quintard et al. (1997) provide suitable information and Fig. 14 plots the values used.

\section{Aknowledgements}

This work has been performed within the framework of the European Project 'HYDRA' (G.A. n. 283797) with financial support of the European Community.

\section{References}

Angeli, P.-E. (2011). Simulation multirésolution et multiéchelle de la thermohydraulique des assemblages de réacteur à neutrons rapides. $\mathrm{PhD}$ thesis, École Centrale Paris.

Angeli, P.-E., Ducros, F., Cioni, O., and Goyeau, B. (2013). Downscaling procedure for convective heat transfer in periodic porous media. Journal of Porous Media, 16(2):123135.

Arbogast, T., Pencheva, G., Wheeler, M. F., and Yotov, I. (2007). A Multiscale Mortar Mixed Finite Element Method. Multiscale Modeling \& Simulation, 6(1):319-346.

Babaei, M. and King, P. R. (2012). A Modified Nested-Gridding for Upscaling-Downscaling in Reservoir Simulation. Transport in Porous Media, 93(3):753-775.

Carbonell, R. G. and Whitaker, S. (1983). Dispersion in pulsed systems-II. Chemical Engineering Science, 38(11):1795-1802.

Chen, Y., Durlofsky, L., Gerritsen, M., and Wen, X. (2003). A coupled local-global upscaling approach for simulating flow in highly heterogeneous formations. Advances in Water Resources, 26(10):1041-1060.

Debenest, G., Mourzenko, V. V., and Thovert, J. F. (2005). Smouldering in fixed beds of oil shale grains: governing parameters and global regimes. Combustion Theory and Modelling, 9(2):301-321. 
Fredrik, B. and Tveito, A. (1998). An upscaling method for one-phase flow in heterogeneous reservoirs. A weighted output least squares (WOLS) approach. Computational Geosciences, 2(110673):93-123.

Gautier, Y., Blunt, M., and Christie, M. (1999). Nested Gridding and Streamline-Based Simulation for Fast Reservoir Performance Prediction. Reservoir Simulation Symposium, 3(1):1-10.

Gray, W. G. (1975). A derivation of the equations for multi-phase transport. Chemical Engineering Science, 30(2):229-233.

Guo, J., Quintard, M., and Laouafa, F. (2015). Dispersion in Porous Media with Heterogeneous Nonlinear Reactions. Transport in Porous Media, 109(3):541-570.

Hou, T. Y. and Wu, X.-H. (1997). A Multiscale Finite Element Method for Elliptic Problems in Composite Materials and Porous Media. Journal of Computational Physics, 134(1):169_ 189.

Jasak, H., Jemcov, A., and Tukovic, Z. (2007). OpenFOAM: A C++ library for complex physics simulations. In International Workshop on Coupled Methods in Numerical Dynamics, Dubrovnik, Croatia.

Jenny, P., Lee, S. H., and Tchelepi, H. A. (2005). Adaptive Multiscale Finite-Volume Method for Multiphase Flow and Transport in Porous Media. Multiscale Modeling \& Simulation, 3(1):50-64.

Juanes, R. (2005). A variational multiscale finite element method for multiphase flow in porous media. Finite Elements in Analysis and Design, 41(7-8):763-777.

Leroy, V., Goyeau, B., and Taine, J. (2013). Coupled Upscaling Approaches For Conduction, Convection, and Radiation in Porous Media: Theoretical Developments. Transport in Porous Media, 97(3):323-347.

Maraun, D., Wetterhall, F., Ireson, a. M., Chandler, R. E., Kendon, E. J., Widmann, M., Brienen, S., Rust, H. W., Sauter, T., Themel, M., Venema, V. K. C., Chun, K. P., Goodess, C. M., Jones, R. G., Onof, C., Vrac, M., and Thiele-Eich, I. (2010). Precipitation downscaling under climate change: Recent developments to bridge the gap between dynamical models and the end user. Reviews of Geophysics, 48(3):1-34.

Marle, C. M. (1967). Ecoulements monophasiques en milieux poreux. Revue de l'Institut Français du Pétrole, 22(10):1467-1509.

Moyne, C. (1997). Two-equation model for a diffusive process in porous media using the volume averaging method with an unsteady-state closure. Advances in Water Resources, 20(2-3):63-76.

Quintard, M. (2014). Transfers in Porous Media. In 15th International Heat Transfer Conference, Kyoto.

Quintard, M., Kaviany, M., and Whitaker, S. (1997). Two-medium treatment of heat transfer in porous media: numerical results for effective properties. Advances in Water Resources, 20(2-3):77-94. 
Quintard, M., Ladevie, B., and Whitaker, S. (2000). Effect of homogeneous and heterogeneous source terms on the macroscopic description of heat transfer in porous media. Energy Engineering, 2(January):482-489.

Quintard, M. and Whitaker, S. (1993). One- and two-equation models for transient diffusion processes in two-phase systems. Advances in Heat Transfer, 23:369.

Quintard, M. and Whitaker, S. (1994a). Transport in ordered and disordered porous media I: The cellular average and the use of weighting functions. Transport in Porous Media, 14(2):163-177.

Quintard, M. and Whitaker, S. (1994b). Transport in ordered and disordered porous media II: Generalized volume averaging. Transport in Porous Media, 14(2):179-206.

Quintard, M. and Whitaker, S. (1994c). Transport in ordered and disordered porous media III: Closure and comparison between theory and experiment. Transport in Porous Media, $15(1): 31-49$.

Quintard, M. and Whitaker, S. (1994d). Transport in ordered and disordered porous media IV: Computer generated porous media for three-dimensional systems. Transport in Porous Media, 15(1):51-70.

Quintard, M. and Whitaker, S. (1994e). Transport in ordered and disordered porous media V: Geometrical results for two-dimensional systems. Transport in Porous Media, 15(2):183196.

Quintard, M. and Whitaker, S. (2000). Theoretical Analysis of Transport in Porous Media. In Vafai, K. and Hadim, H. A., editors, Handbook of Heat Transfer in Porous Media, chapter 1, pages 1-52. Marcel Dekker, Inc., New York.

Quintard, M. and Whitaker, S. (2005). Coupled, Nonlinear Mass Transfer and Heterogeneous Reaction in Porous Media. In Vafai, K., editor, Handbook of Porous Media, chapter 1. Taylor \& Francis Group, New York, 2nd editio edition.

Von Storch, H., Zorita, E., and Cubasch, U. (1993). Downscaling of global climate change estimates to regional scales: An application to Iberian rainfall in wintertime. Journal of Climate, 6(6):1161-1171.

Whitaker, S. (1969). Advances in Theory of Fluid Motion in Porous Media. Industrial and Engineering Chemistry, 61(12):14.

Whitaker, S. (1986). Flow in porous media I: A theoretical derivation of Darcy's law. Transport in porous media, 1(1):3-25.

Whitaker, S. (1996). The Forchheimer equation: a theoretical development. Transport in Porous media, 25(1):27-61.

Whitaker, S. (1998). Coupled transport in multiphase systems: a theory of drying. Advances in Heat Transfer, 31:1-104.

Whitaker, S. (1999). The Method of Volume Averaging. Kluwer Academic Publishers, Dordrecht. 
Wilby, R. L., Wigley, T. M. L., Conway, D., Jones, P. D., Hewitson, B. C., Main, J., and Wilks, D. S. (1998). Statistical downscaling of general circulation model output: A comparison of methods. Water Resources Research, 34(11):2995. 
Table 1: Numerical values for the reference problem

\begin{tabular}{cccl}
\hline Quantity & Symbol & Value & \multicolumn{1}{c}{ Unit } \\
\hline Cell size & $r_{0}$ & 0.1 & $\mathrm{~m}$ \\
Cylinder radius & $r_{\mathrm{c}}$ & 0.04 & $\mathrm{~m}$ \\
Porosity & $\varepsilon$ & 0.497 & - \\
Specific surface area & $A$ & 25.1 & $\mathrm{~m}^{-1}$ \\
Dynamic viscosity & $\mu$ & $1 \times 10^{-5}$ & $\mathrm{Pas}$ \\
Density & $\rho$ & 1 & $\mathrm{~kg} \mathrm{~m}^{-3}$ \\
Heat capacity & $c_{p}$ & $1 \times 10^{3}$ & $\mathrm{~J} \mathrm{~kg}^{-1} \mathrm{~m}^{-3}$ \\
Heat conductivity & $k$ & $1 \times 10^{-2}$ & $\mathrm{~W} \mathrm{~m}^{-1} \mathrm{~K}^{-1}$ \\
Kinematic viscosity & $v$ & $1 \times 10^{-5}$ & $\mathrm{~m}^{2} \mathrm{~s}^{-1}$ \\
Heat diffusivity & $a$ & $1 \times 10^{-5}$ & $\mathrm{~m}^{2} \mathrm{~s}^{-1}$ \\
Prantl number & $\mathrm{Pr}$ & 1 & - \\
\hline
\end{tabular}

Table 2: Numerical values for the reference problem

\begin{tabular}{cccl}
\hline Quantity & Symbol & Value & Unit \\
\hline Cell size & $r_{0}$ & 0.1 & $\mathrm{~m}$ \\
Cylinder radius & $r_{\mathrm{c}}$ & 0.0445 & $\mathrm{~m}$ \\
Porosity & $\varepsilon$ & 0.377 & - \\
Specific surface area & $A$ & 28.0 & $\mathrm{~m}^{-1}$ \\
\hline
\end{tabular}

